\title{
Discovery of a variable lead-rich hot subdwarf: UVO $0825+15$
}

\author{
C. S. Jeffery, ${ }^{1,2 \star}$ A. S. Baran, ${ }^{3}$ N. T. Behara,${ }^{1}$ A. Kvammen, ${ }^{4}$ P. Martin,${ }^{1,2}$ \\ Naslim N., ${ }^{5,6}$ R. H. Østensen, ${ }^{7}$ H. P. Preece, ${ }^{1,8}$ M. D. Reed, ${ }^{7}$ J. H. Telting, ${ }^{4}$ \\ and V. M. Woolf ${ }^{9}$ \\ ${ }^{1}$ Armagh Observatory and Planetarium, College Hill, Armagh BT61 9DG \\ ${ }^{2}$ School of Physics, Trinity College Dublin, College Green, Dublin 2, Ireland \\ ${ }^{3}$ Suhora Observatory and Kraków Pedagogical University, ul. Podchorązych 2,30-084,Kraków, Poland \\ ${ }^{4}$ Nordic Optical Telescope, Rambla José Ana Fernández Pérez 7, 38711 Breña Baja, Spain \\ ${ }^{5}$ Academia Sinica Institute of Astronomy and Astrophysics, Taipei 10617, Taiwan, Republic of China \\ ${ }^{6}$ National Astronomical Observatory of Japan, 2-21-1 Osawa, Mitaka, Tokyo 181-8588, Japan \\ ${ }^{7}$ Department of Physics, Astronomy and Materials Science, Missouri State University, 901 S. National, Springfield, MO 65897, USA \\ ${ }^{8}$ Institute of Astronomy, University of Cambridge, Madingley Rd., Cambridge, CB3 0HA, UK \\ ${ }^{9}$ Department of Physics, University of Nebraska at Omaha, 6001 Dodge Street, Omaha, NE 68182, USA
}

Accepted ..... Received .....; in original form .....

\begin{abstract}
UVO $0825+15$ is a hot bright helium-rich subdwarf which lies in K2 Field 5 and in a sample of intermediate helium-rich subdwarfs observed with Subaru/HDS. The K2 light curve shows low-amplitude variations, whilst the Subaru spectrum shows Pbiv absorption lines, indicative of a very high lead overabundance. UVO $0825+15$ also has a high proper motion with kinematics typical for a thick disk star. Analyses of ultraviolet and intermediate dispersion optical spectra rule out a short-period binary companion, and provide fundamental atmospheric parameters of $T_{\text {eff }}=38900 \pm 270 \mathrm{~K}$, $\log g / \mathrm{cm} \mathrm{s}^{-2}=5.97 \pm 0.11, \log n_{\mathrm{He}} / n_{\mathrm{H}}=-0.57 \pm 0.01, E_{B-V} \approx 0.03$, and angular radius $\theta=1.062 \pm 0.006 \times 10^{-11}$ radians (formal errors). The high-resolution spectrum shows that carbon is $>2$ dex subsolar, iron is approximately solar and all other elements heavier than argon are at least $2-4$ dex overabundant, including germanium, yttrium and lead. Approximately 150 lines in the blue-optical spectrum remain unidentified. The chemical structure of the photosphere is presumed to be determined by radiatively-dominated diffusion. The K2 light curve shows a dominant period around $10.8 \mathrm{~h}$, with a variable amplitude, its first harmonic, and another period at $13.3 \mathrm{~h}$. The preferred explanation is multi-periodic non-radial oscillation due to g-modes with very high radial order, although this presents difficulties for pulsation theory. Alternative explanations fail for lack of radial-velocity evidence. UVO $0825+15$ represents the fourth member of a group of hot subdwarfs having helium-enriched photospheres and 3-4 dex overabundances of trans-iron elements, and is the first lead-rich subdwarf to show evidence of pulsations.
\end{abstract}

Key words: stars: chemically peculiar, stars: oscillations, stars: variables: general, stars: early-type, stars: individual: UVO $0825+15$

\section{INTRODUCTION}

The theoretical helium "main-sequence" is defined by stars consisting almost entirely of helium, and converting helium to carbon at their centres via the nuclear triple-alpha process, which form a continuum that depends primarily upon

^ E-mail: csj@arm.ac.uk the mass of the star. There exists a diverse population of low-mass hot stars which have surface properties placing them close to this sequence. They are commonly referred to as "hot subdwarfs", since their surfaces are between 4 and 8 times hotter than that of the Sun, and they lie below the more familiar hydrogen main-sequence, where stars are referred to as "dwarfs" to distinguish them from the larger giants and supergiants. In contrast to the sur- 
faces of hydrogen main-sequence stars, which persistently show a helium:hydrogen ratio of around 1:9 (by number), hot subdwarfs show considerable diversity with surface helium:hydrogen ratio ranging from the extremely helium-poor subdwarf B (sdB) stars (1:10000), extremely helium-rich subdwarf O (He-sdO) stars (99:1), and a small number of intermediate composition $(1: 9-9: 1)$. This diversity reflects a variety of evolutionary origin evidenced by various duplicities, with sdB stars having white dwarf, M dwarf, F-G dwarf, or planetary companions, or none. Progress in understanding these origins has been assisted by the discovery of pulsations in both sdB and sdO stars, although these are not universal. A recent review examines many of the properties of hot subdwarfs, and the problems surrounding them (Heber 2016).

The helium-rich subdwarfs themselves represent a heterogeneous group having a wide range in surface temperature $(25000-45000 \mathrm{~K})$ (Naslim et al. 2010), including at least one spectroscopic binary (Naslim et al. 2012), and a small group with extraordinary $(3-4$ dex $)$ overabundances of germanium, strontium, yttrium, zirconium, and lead (Naslim et al. 2011, 2013). It has been assumed that radiative levitation selectively lifts certain species into the lineforming region in the atmospheres of these stars; a proof that this explanation is correct is still wanting. The prototype of these "heavy-metal stars", the zirconium star LS IV $-14^{\circ} 116$ pulsates with multiple periods of around 1800 s (Ahmad \& Jeffery 2005; Green et al. 2011). No instability mechanism has yet been identified by which to drive these pulsations.

This paper is concerned with two questions; 1) evidence for pulsations in hot subdwarfs which fall within the fields observed by the spacecraft $K 2$ and 2) the origin of the class of intermediate helium-rich subdwarfs. By coincidence, the same star was chosen to address each of these questions with observations carried out during 2015 in K2 (Campaign 5) and in a high-resolution spectroscopic survey with the Subaru telescope on Mauna Kea.

The star, known variously as TD1 $31206=$ TD1 32707 (Thompson et al. 1978) = UVO 0825+15 (Berger \& Fringant 1980) $=$ [CW83 $]$ 0825+15 (Carnochan \& Wilson $1983)=$ TYC 808-490-1 = GALEX J082832.8+145205 = 2MASS J08283287+1452024 = EPIC 211623711, was classified "sdO" by Berger \& Fringant (1980) and Kilkenny (1988). The equatorial coordinates for epoch J2000 and equinox 2000 are $\alpha=08 h 28 m 32.876 s, \delta=+14^{\circ} 52^{\prime} 02.51^{\prime \prime}$. The SIMBAD database (Wenger et al. 2000) does not associate UVO $0825+15$ with that identified by other synonyms due to low positional precision. The star exhibits a substantial proper motion, exceeding 24 mas y $^{-1}$ (Høg et al. 2000).

Observations with GALEX and the NTT were used by Vennes et al. (2011) to derive an effective temperature $T_{\text {eff }}=36650 \pm 650 \mathrm{~K}$, a surface gravity $\log \mathrm{g} / \mathrm{cm} \mathrm{s}^{-2}=5.65 \pm$ 0.14 , and a surface helium-to-hydrogen ratio $\log n_{\mathrm{He}} / n_{\mathrm{H}}=$ $-0.50 \pm 0.08$. Németh et al. (2012) used low-dispersion optical spectra to obtain $T_{\text {eff }}=37060 \pm 600 \mathrm{~K}, \log g / \mathrm{cm} \mathrm{s}^{-2}=$ $5.92 \pm 0.10$, and $\log n_{\mathrm{He}} / n_{\mathrm{H}}=-0.62 \pm 0.08$.

In the following, we describe the observations ( $(2)$, the derivation of photospheric properties ( $(3)$ and discovery of strong lead absorption lines ( $\$ 4)$, the discovery of light variability, analysis of the light curve and its interpretation ( $(5)$, and analysis of the kinematics (§6). We compare these properties with those of other helium-rich subdwarfs $(\S 7)$.
Table 1. Relative radial velocities of UVO $0825+15$ from NOT/ALFOSC spectroscopy.

$\begin{array}{lrr}\text { BJD } & \delta v & \pm \\ 2457436.3442983 & 9.94 & 3.62 \\ 2457443.5281802 & -12.27 & 5.64 \\ 2457444.5318080 & 5.36 & 3.94 \\ 2457447.5096600 & -9.54 & 5.05 \\ 2457447.5833238 & -12.28 & 4.41 \\ 2457449.4176574 & -5.46 & 4.77\end{array}$

\section{OBSERVATIONS}

\subsection{Spectrophotometry: IUE and BVRJHK}

As an ultraviolet bright source identified in the TD1A S2/68 ultraviolet sky survey (Carnochan \& Wilson 1983), UVO $0825+15$ was an early target for the International Ultraviolet Explorer (IUE). Images LWR09914 and SWP11306 were obtained using both small and large apertures on 1981, February 14. We have used the large-aperture images for photometric reliability, supplemented by the small-aperture data in the range $1200-1400 \AA$ where many large-aperture pixels are saturated.

In addition there exists photometry in broad-band filters. From the SIMBAD database, $B=11.57 \pm 0.10$, $V=11.82 \pm 0.21, R=12.01 \pm 0.04, J=12.425 \pm 0.021$, $H=12.586 \pm 0.026$, and $K=12.652 \pm 0.029 \mathrm{mag}(\mathrm{H} \varnothing \mathrm{g}$ et al. 2000; Zacharias et al. 2009; Cutri et al. 2003).

\subsection{Nordic Optical Telescope/ALFOSC spectroscopy}

We obtained 6 low resolution spectra between 2016 February 17 and March 1 using the 2.56-m Nordic Optical Telescope with ALFOSC, grism \#18 and a 0.5" slit, with exposure times of $150-200 \mathrm{~s}$. The spectra have $R \approx 2000$ (resolution element $2.2 \AA$ ) and $\mathrm{S} / \mathrm{N}$ ranging from 55 to 130 .

The data were homogeneously reduced and analysed. Standard reduction steps within IRAF include bias subtraction, removal of pixel-to-pixel sensitivity variations, optimal spectral extraction, and wavelength calibration based on arc-lamp spectra. The target spectra and the mid-exposure times were shifted to the barycentric frame of the solar system.

Radial velocities were derived with the FXCOR package in IRAF. We used the $\mathrm{H} \beta, \mathrm{H} \gamma, \mathrm{H} \delta, \mathrm{H} \zeta$ and $\mathrm{H} \eta$ lines to determine the radial velocities (RVs), and used the normalised average spectrum as a template. The final RVs were adjusted for the position of the target in the slit, judged from slit images taken just before and just after the spectral exposure.

The relative velocities are shown in Table 1, where the root mean square deviation around the mean of $8.7 \mathrm{~km} \mathrm{~s}^{-1}$ is less than twice the mean standard error $\left(4.6 \mathrm{~km} \mathrm{~s}^{-1}\right.$, individual errors from FXCOR). With six measurements obtained over 2 weeks, this rules out any short-period $(<7 \mathrm{~d})$ binary companion unless the inclination is very small.

\section{$2.3 \quad$ Subaru/HDS spectroscopy}

UVO $0825+15$ was observed with the High Dispersion Spectrograph (HDS) (Noguchi et al. 2002) on the Subaru telescope, operating in service mode, on 2015 June 3. Two observations were made consecutively, each with exposure time 
$240 \mathrm{~s}$. A slit width of $0.4 \mathrm{~mm}$ was used, corresponding to a projected resolution $R=45000$. The data were reduced using standard IRAF procedures, together with the reduction manual and scripts written by Subaru staff for reducing HDS spectra (Aoiki 2008; Tujitsu 2013). Wavelengths were corrected for earth motion, the two observations were combined, and the orders were merged to provide a single rectified spectrum. Instrumental artefacts resembling broad aborption lines with emission wings (or vice versa) occur in a number of places. These were clipped or masked from the spectrum during analysis.

The radial velocity for the combined order-merged spectrum was measured using cross-correlation against two theoretical spectra of differing $T_{\text {eff }}$ and metallicity and using two spectral ranges, one in each part of the spectrogram. These four measurements gave a mean radial velocity of $+56.4 \pm 0.5 \mathrm{~km} \mathrm{~s}^{-1}$.

\section{$2.4 \quad K 2$ photometry}

Short cadence K2 observations, providing one image every 58.85 seconds, were obtained between 2015 April 27 and 2015 July 10. The K2 mission only uses two gyros for stability and so the spacecraft rolls from solar pressure, requiring occasional thruster firings for pointing corrections. Consequently, objects drift across an array of pixels and unequal pixel sensitivity produces fluxes which are pixel-correlated. To correct for this we have developed a processing pipeline using a combination of IRAF photometry packages and custom decorrelation routines. We downloaded pixel files from the Mikulski Archive for Space Telescopes and used DAOFIND to determine the motion of the stellar profile. We then extracted fluxes using aperture photometry and iteratively decorrelated the fluxes in both directions of the pixel array. The stellar signal has three distinct regions and because the first two are so strong, we found it necessary to prewhiten the stellar signal prior to determining pixel-flux correlations for those regions. Pixel-correlated fluxes were then subtracted from the light curve, effectively removing motion-induced variations. The light curve was then sigma clipped to remove outliers and spline fitted to remove trends on the order of six days and longer. The resulting light curve is shown in Fig. 1, where K2 times are defined as barycentric Julian Date (BJD) - 2454833.0.

\section{SPECTROSCOPIC ANALYSIS}

\subsection{Model Atmospheres}

The analysis of stellar spectra depends heavily on the computation of physically realistic models for the temperature and density structure of the stellar atmosphere and for the detailed spectrum of radiation emergent therefrom. For the majority of this study we have computed bespoke models for both using Armagh codes STERNE (Behara \& Jeffery 2006) and SPECTRUM ${ }^{1}$, respectively.

STERNE assumes a plane-parallel atmosphere in radiative, hydrostatic and local-thermodynamic equilibrium (LTE). Monochromatic continuous opacities are obtained

1 www.arm.ac.uk/ csj/software_store/guide/spectrum.html from the Opacity and Iron Projects (The Opacity Project Team 1995; Hummer et al. 1993, et seq.) for up to six ions of all elements hydrogen through silicon, sulphur, argon, calcium and iron. Line blanketing is treated by Opacity Sampling in a list of 559000 absorption lines (including all species from hydrogen to bismuth, thorium and uranium). Use of this code allows the speed and flexibility necessary to produce multiple model grids of arbitrary composition. Since the input composition needs to match the measured composition of the star, which is not known a priori, some iterations are necessary to obtain a final solution. We note that STERNE differs from many "LTE" codes by allowing for electron scattering in the radiative transfer equation. The full LTE approximation, which equates the source function to the mean intensity and to the Planck function (i.e. $S_{\nu}=J_{\nu}=B_{\nu}$ ), breaks down at high temperature and low surface gravities. STERNE also provides emergent fluxes $\left(F_{\lambda}\right)$ at low resolution from $220 \AA$ to $20 \mu \mathrm{m}$.

SPECTRUM is a formal solution code providing a highresolution emergent spectrum for a given input model atmosphere. For consistency, it must use the same continuous opacity and equation of state as used for the input model. Line profiles for metal lines are computed using Voigt profiles, including thermal, micro-turbulent, radiative, electron and van der Waal's broadening, where appropriate line data exist. Stark broadened profiles for $\mathrm{H}, \mathrm{He}^{0}$ and $\mathrm{He}^{+}$lines are computed using tables from Vidal et al. (1973), Beauchamp et al. (1997) and Schöning \& Butler (1989).

\subsection{Effective temperature from spectrophotometry}

The most reliable way to measure the effective temperature of a hot star is to use the total-flux method. Ideally the observed bolometric flux $\left(f=L / 4 \pi d^{2}\right)$ is obtained by integrating over all wavelengths, the angular diameter $(\theta=r / d)$ is obtained by normalising the fluxes at some reference wavelength to the fluxes of a model stellar atmosphere with similar effective temperature, and the effective temperature deduced by eliminating $d$ to obtain

$$
\sigma T_{\text {eff }}^{4}=\left(f 4 \pi d^{2} / 4 \pi r^{2}\right)=\left(f / \theta^{2}\right),
$$

where $\sigma$ is the Stefan-Boltzmann constant. Where significant parts of the overall flux distribution lie outside the observed wavelength range, these can be supplied by reference to a suitable model stellar atmosphere. The observed fluxes must also be corrected for any interstellar extinction (dereddened). Effectively, the flux integral and dereddening can be substituted by finding the model atmosphere and extinction curve which best fits the observed fluxes, and deducing $T_{\text {eff }}$ and the colour excess $E_{B-V}$ therefrom.

There are hazards in this approach: i) the flux distribution (not the total flux) also depends on the surface gravity and composition and ii) for hot stars, the effects of extinction and temperature on the gradient of the ultraviolet continuum are partially degenerate, except around the broad interstellar $2175 \AA$ absorption feature. Fortunately, in the case of UVO $0825+15$, there is no evidence for any feature at $2175 \AA$, so we initially adopted $E_{B-V} \approx 0$. So it only remains to establish appropriate values for surface gravity and composition. This is an iterative process which requires information from spectroscopy. 


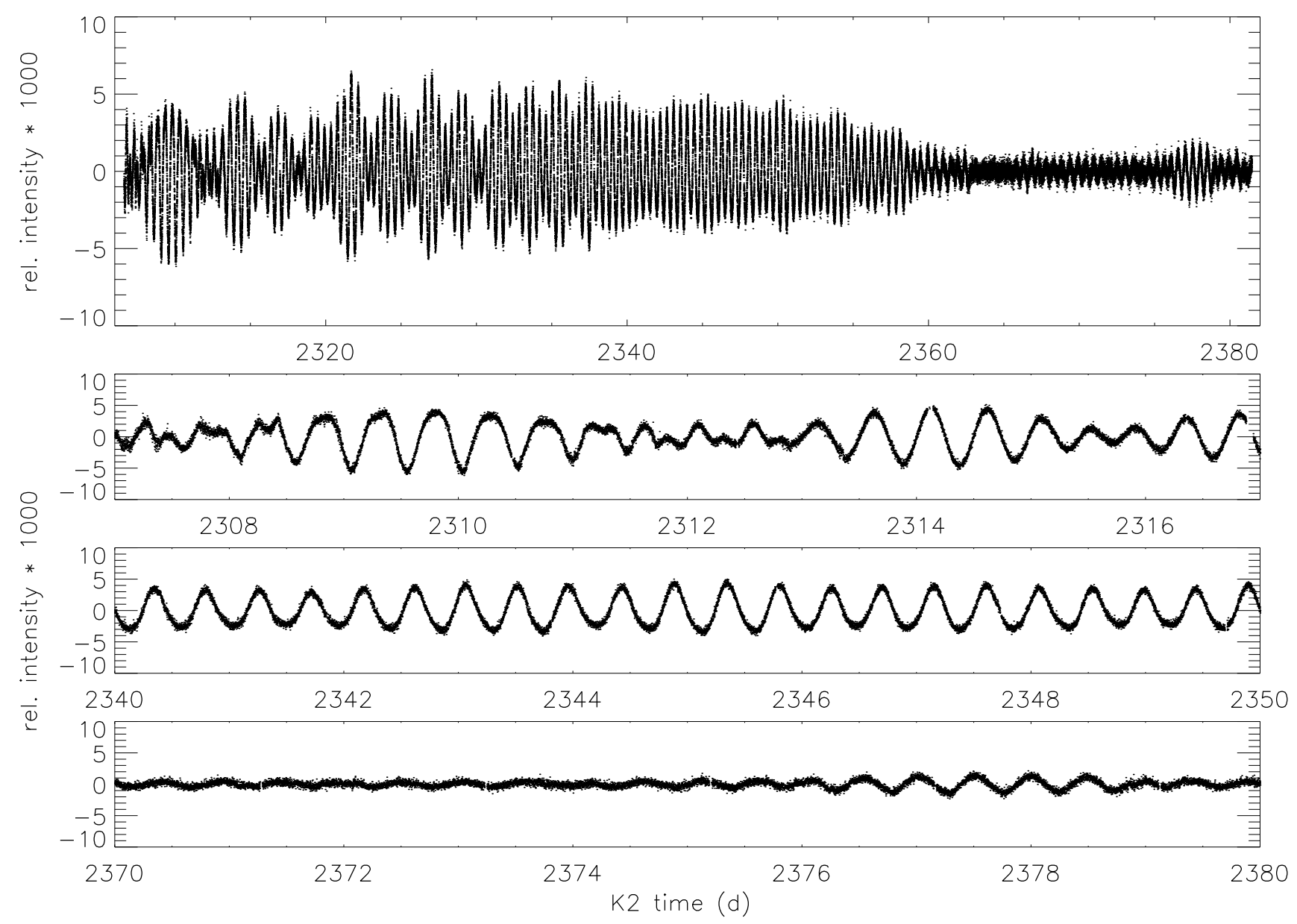

Figure 1. K2 short cadence light curve of UVO 0825+15 showing the entire dataset from Campaign 5 (top) and three 10-day segments (below) displaying different characteristics.

As a first approximation we computed a grid of lineblanketed model atmospheres with $\mathrm{H}: \mathrm{He}$ ratios in the range 100:00, 90:10, 70:30, 50:50, 30:70, 10:90, 1:99, and 00:100 (labelled $\mathbf{h} x \mathbf{h e} y y$, where $x x$ and $y y$ correspond to the hydrogen:helium number fractions per cent just described). The chemical composition due to all other elements was taken to be solar by number (labelled p00). The micro-turbulent velocity was fixed at $v_{t}=5 \mathrm{~km} \mathrm{~s}^{-1}$, typical for B-type stars. A test with a small model grid having $v_{t}=2 \mathrm{~km} \mathrm{~s}^{-1}$ showed no discernible difference in the theoretical flux distribution, or in the photospheric solution discussed below.

The intermediate-dispersion spectroscopy (§3.3) indicates $\log g / \mathrm{cm} \mathrm{s}^{-2} \approx 6$ and $n_{\mathrm{H}}: n_{\mathrm{He}} \approx 80: 20$, so we initially used the grid of atmospheres identified as h70he30p00/t...g600p00, where t... represents $T_{\text {eff }}$ in the grid $(28, \ldots(2) \ldots, 42,45) \mathrm{kK}$.

Applying our $\chi^{2}$-minimization method (FFIT: Jeffery et al. 2001) to the $I U E+B V R J H K$ spectrophotometry described in $\S 2.1$, and after iterating with $\S 3.3$, we were unable to find a unique solution for $T_{\text {eff }}, E_{B-V}$ and $\theta$. Fig. 2 demonstrates that for this star, the overall flux distribution is insensitive to $T_{\text {eff }}$ within the optical and $I U E$ range, with only the detailed line spectrum at high-resolution being af- fected. It will be necessary to make ultraviolet observations at shorter wavelength or at higher resolution in order to break this degeneracy. We also note that the models show insufficient absorption around $\lambda 1215 \AA$. This is due to the omission of Stark broadening from the He II series in the calculation of the model atmosphere; the hydrogen Ly $\alpha$ line is very weak at the $T_{\text {eff }}$ of the solution. This omission and the use of only 559034 strong lines in the input line list introduces a small systematic error to the predicted flux distribution. However this error is small compared with the effects of both line and continuous far-ultraviolet opacity due to other ions, such as calcium, when significantly overabundant.

Consequently, it was necessary to adopt $T_{\text {eff }}=39000 \mathrm{~K}$ on the basis of medium-resolution optical spectroscopy (§3.3). The procedure was repeated after obtaining a first estimate for the surface composition (cf. $\S 4$ ) and computing a model grid based on this mixture (labelled h80he20_uvo0825). Some experimentation showed that $E_{B-V}$ could not be exactly zero; a value of 0.03 was obtained by inspection. $\theta=1.062 \pm 0.006 \times 10^{-11} \mathrm{rad}$ is given by fitting the model to the observations (Fig. 2). The latter is subject to a systematic error of $\pm 10 \%$ arising from the possibility of unknown extreme- and far-ultraviolet opac- 


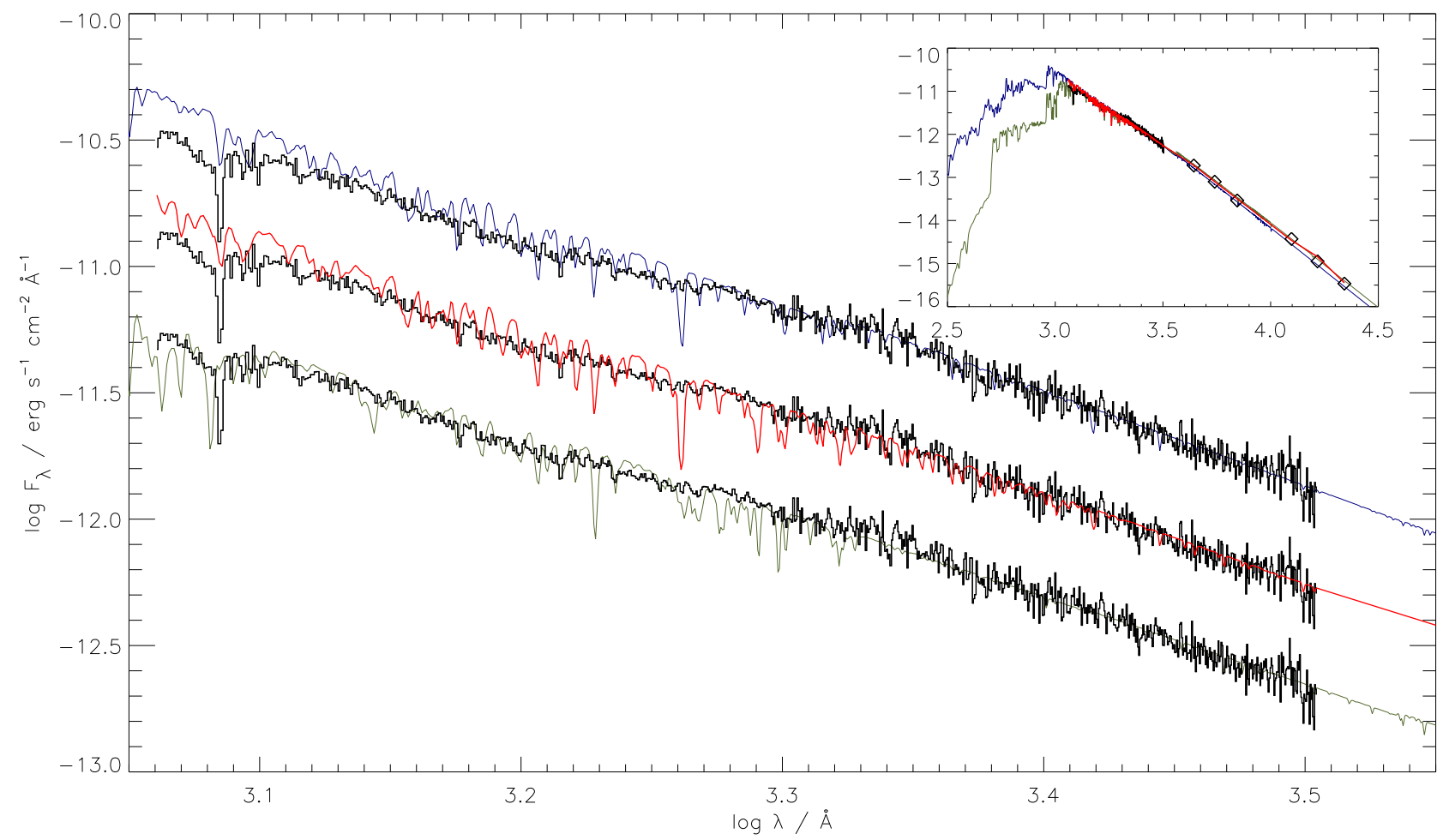

Figure 2. The merged IUE spectrum of UVO $0825+15$ (black histogram) compared with a theoretical spectrum (red), with properties matched to those obtained from optical intermediate and high-resolution spectroscopy, with $T_{\text {eff }}=39000 \mathrm{~K}, \log g / \mathrm{cm} \mathrm{s}^{-2}=6$, from grid h80he20_uvo 0825. $E_{\mathrm{B}-\mathrm{V}}=0.03$ was found by inspection and $\theta=1.062 \pm 0.006 \times 10^{-11}$ radians is obtained by $\chi^{2}-$ minimization. The fit is resampled to the wavelength grid of the observations and convolved with an instrumental profile having full-width half-maximum of $7 \AA$. Model atmospheres with $T_{\text {eff }}=30000$ and $45000 \mathrm{~K}$ are shown in green and blue and displaced down and up by 0.4 dex, respectively, also convolved with the instrumental profile, and normalized to the $39000 \mathrm{~K}$ solution at $V$. The inset panel shows the same data extended to the far ultraviolet and to the infrared, with $B V R J H K$ magnitudes shown as diamonds.

ity arising from abundant species $(Z>26)$ not included in the model (i.e. not included in either the Opacity or Iron Projects).

\subsection{Surface gravity and hydrogen:helium ratio from $N O T / A L F O S C$ spectroscopy}

A second option for securing $T_{\text {eff }}$ and, in addition, $\log g$, and $n_{\mathrm{He}} / n_{\mathrm{H}}$, is to use the profiles of hydrogen, and neutral and ionized helium lines in the blue-optical spectrum. The widths of these lines in hot stars are sensitive to pressure and hence to $\log g ; n_{\mathrm{He}} / n_{\mathrm{H}}$ can be measured from the relative strength of hydrogen and helium lines; $T_{\text {eff }}$ can be measured from the neutral-to-ionized helium ratio as well as from the Balmer decrement.

Our approach was to seek an optimum fit to the mean NOT/ALFOSC spectrum of UVO $0825+15$ using $\chi^{2}$ minimisation in a grid of models as follows. The entire wavelength region between 3680 and $5150 \AA$ was used. The observed spectrum was re-normalized prior to each $\chi^{2}$ minimisation using a pseudo continuum obtained as follows. Regions of spectrum deemed to be free from both observed and predicted absorption lines were identified as pseudocontinuum regions. A predicted spectrum, resampled onto the wavelength grid of the observed spectrum, was divided throughout by the latter. The errors associated with each wavelength in the pseudo-continuum regions of the observed spectrum were associated with the ratioed spectrum. Elsewhere, the errors were set to $10^{10}$. The ratioed spectrum was smoothed, using the inverse of the associated errors to weight individual data points and by convolution with a Gaussian having full-width half-maximum of $200 \AA$. The observed spectrum was multiplied by this smoothed pseudocontinuum to obtain a re-normalized spectrum. The object was to ensure a satisfactory fit even in regions of spectrum where overlap between broad lines means there is no true continuum, and also to ensure that the renormalisation does not affect the local profile of any individual absorption line. A $\chi^{2}$-minimisation procedure using a Levenburg-Marquardt algorithm (Press et al. 1989), as implemented in the package SFIT (Jeffery et al. 1998), was used to solve for $T_{\text {eff }}, \log g$, $n_{\mathrm{He}} / n_{\mathrm{H}}$, and the velocity shift $v$. Being the velocity-shifted mean of several spectra having different heliocentric velocities, the latter had no physical meaning for this spectrum.

Re-normalisation was iterated to convergence with the final solution, with convergence represented by changes in successive solutions becoming smaller than one tenth the formal errors. The final re-normalised spectrum and best-fit theoretical spectrum are shown in Fig. 3, with principal H, HeI and HeII lines identified, these providing the primary constraints on $T_{\text {eff }}, \log g$, and $n_{\mathrm{He}} / n_{\mathrm{H}}$. The adopted continuum windows are also identified in Fig. 3. 


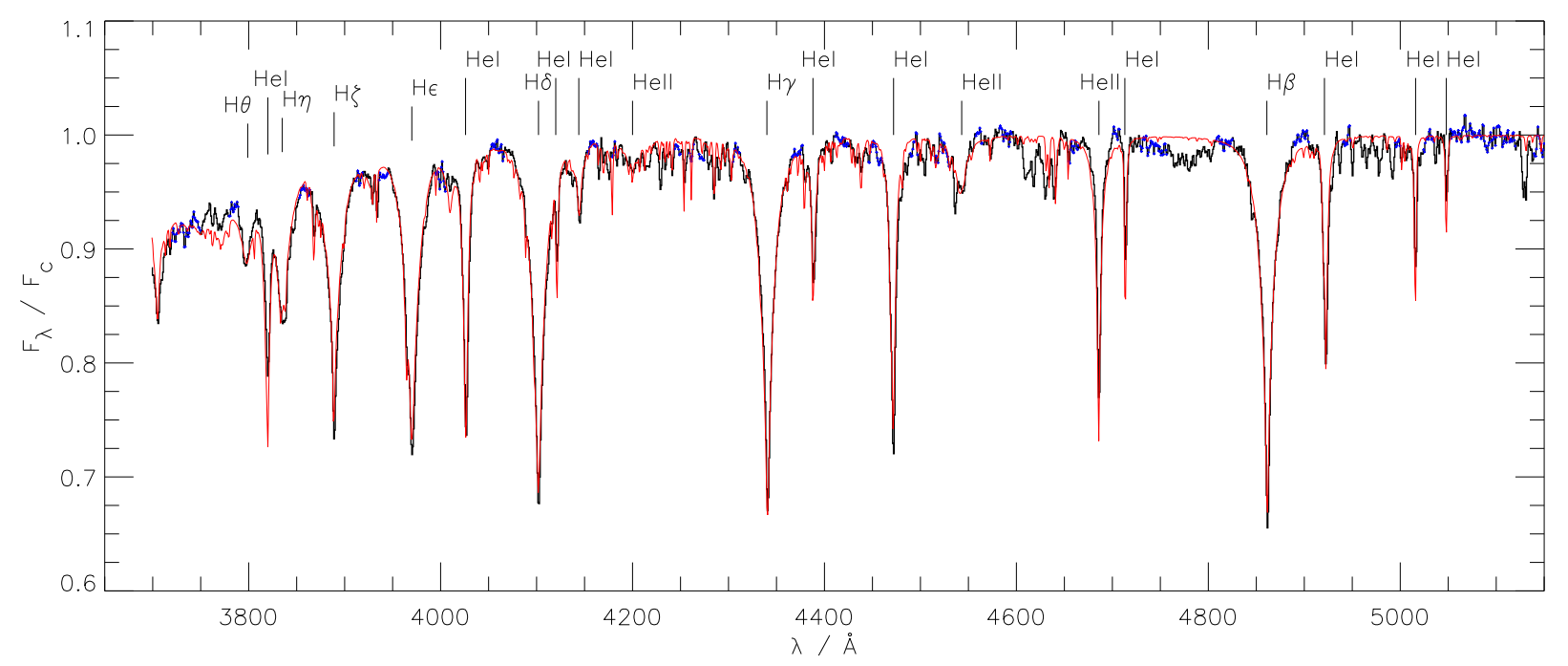

Figure 3. The mean NOT/ALFOSC spectrum of UVO $0825+15$ (black histogram) compared with the best-fit spectrum (red), obtained from a grid of line-blanketed model atmospheres with composition tailored to the observations and having the solution $T_{\text {eff }}=38900 \pm$ $270 \mathrm{~K}, \log g / \mathrm{cm} \mathrm{s}^{-2}=5.97 \pm 0.11, n_{\mathrm{He}}=0.21 \pm 0.05\left(\log n_{\mathrm{He}} / n_{\mathrm{H}}=-0.6 \pm 0.01\right)$. Pseudo-continuum points used for re-normalisation are marked in blue.

Using a grid of models with solar metallicity composition (p00) and (H:He) number fractions (0.9:0.1), (0.7:0.3), and (0.5:0.5), we obtained $T_{\mathrm{eff}}=39100 \pm 250 \mathrm{~K}$, $\log g / \mathrm{cm} \mathrm{s}^{-2}=6.02 \pm 0.12$ and $n_{\mathrm{He}}=0.21 \pm 0.05$ $\left(\log n_{\mathrm{He}} / n_{\mathrm{H}}=-0.57 \pm 0.01\right)$ The errors are formal statistical errors in the fit.

Changing the initial values in the multi-parameter fit (e.g. from below the final solution to above the final solution) had no effect on the result. Small changes in the definition of continuum windows led to systematic errors of $\delta T_{\text {eff }} \approx \pm 100 \mathrm{~K}, \delta \log g \approx \pm 0.1$ and $\delta \log n_{\mathrm{He}} / n_{\mathrm{H}} \approx \pm 0.02$. Reducing the width of the Gaussian used to smooth the continuum to $50 \AA$ (from $200 \AA$ ) provided some benefits for continuum fitting at the possible expense of allowing the fit to lead the final solution, in this case by $\delta T_{\text {eff }}=-190 \mathrm{~K}$, $\delta \log g=+0.15$ and $\delta \log n_{\mathrm{He}} / n_{\mathrm{H}}=-0.03$.

The initial assumption of a solar-mixture of metals is inconsistent with the observations; some lines appeared in either the observation or the model which do not appear in the other. This inconsistency was partially resolved following the fine-abundance analysis described in $\S 4$ and Table 2 and the computation of a grid of models with fixed helium abundance $\left(n_{\mathrm{H}}: n_{\mathrm{He}}=80: 20\right)$ approximating the star's surface composition (h80he20_uvo0825). Repeating $\chi^{2}$ minimization and re-normalisation led to a final solution with $T_{\text {eff }}=38900 \pm 270 \mathrm{~K}$ and $\log g / \mathrm{cm} \mathrm{s}^{-2}=5.97 \pm 0.11$ (formal errors).

These results give $\delta T_{\text {eff }}=2500 \mathrm{~K}$ and $\delta \log g=0.35$ higher than, and $\log n_{\mathrm{He}} / n_{\mathrm{H}}$ similar to those given by Vennes et al. (2011); Németh et al. (2012) obtained a surface gravity and helium abundance similar to the new measurement, but with $T_{\text {eff }}$ more similar to that of Vennes et al. (2011). The Vennes et al. (2011) result was based on nonLTE models containing hydrogen and helium only, with no allowance for back-warming due to opacity from other elements, or to the blanketing effects due to ultraviolet metal lines, which we have already shown to be significant (Fig. 2). Németh et al. (2012) included more species in their cus- tomized non-LTE model atmosphere calculation, but were only able to measure the abundance of one element, nitrogen, with upper limits for carbon and oxygen.

In order to assess the impact of the LTE approximation, a non-LTE model atmosphere was obtained using the Tübingen Model Atmosphere Package (TMAP) at the German Astrophysical Virtual Observatory (Werner et al. 2012; Ringat et al. 2012) ${ }^{2}$. The parameters were $T_{\text {eff }}=40000 \mathrm{~K}, \log \mathrm{g} / \mathrm{cm} \mathrm{s}^{-2}=6.0$, and composition approximately matched to that of the h80he20_uvo0825 grid. The model included ions of hydrogen (2 ions), helium (3), carbon (5), nitrogen (5), oxygen (5), neon (5), sodium (5) and magnesium (5), represented by a total of 1435 LTE levels and 486 non-LTE levels. The formal solution for the blue-optical spectrum was convolved with a $1 \AA$ Gaussian and rebinned at $0.4 \AA$, in order to match as closely as possible the NOT/ALFOSC spectrum. Using SFIT and the h80he20_uvo0825 grid, a best-fit match to the TMAP model was sought in the same way as before, yielding $T_{\text {eff }}=40740 \pm 130 \mathrm{~K}$ and $\log g / \mathrm{cm} \mathrm{s}^{-2}=6.18 \pm 0.06$. This is only an indication of the systematic errors; whilst the TMAP model drops the approximation of LTE, it also omits a substantial amount of line opacity in the ultraviolet, with consequences for the temperature structure of the photosphere.

\section{ABUNDANCE ANALYSIS}

A first inspection of the Subaru/HDS spectrum of UVO $0825+15$ revealed the presence of several strong lines due to triply ionized lead (Fig. 4), one of the defining characteristics of this star and one demanding an urgent and detailed abundance analysis.

A major difficulty with the analysis of échelle spectra of high-gravity hot stars is the calibration of the flux, and

2 http://dc.g-vo.org/theossa 

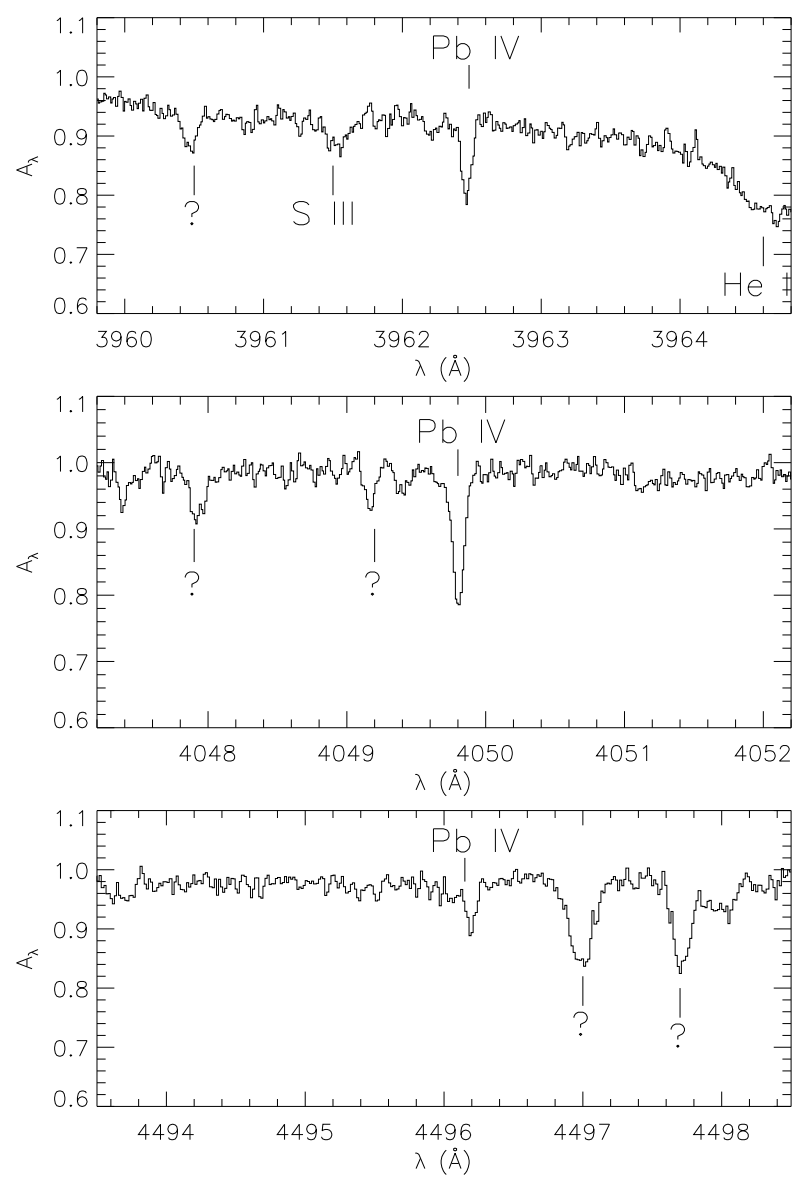

Figure 4. Absorption lines dues to triply-ionized lead in the Subaru HDS spectrum of UVO $0825+15$. The spectrum has been shifted to the laboratory rest-frame. Identified lines are marked by ion; unidentified lines are marked '?'.

particularly the definition of the continuum, due to the extent of the broad Balmer and helium absorption lines over entire and adjacent échelle orders. Thus it is risky to rely heavily on atmospheric parameters derived solely from such lines. However, in the current case, the NOT/ALFOSC spectrum provides a reference, and enables a robust approach to measuring the surface abundances in UVO $0825+15$.

The process essentially involves renormalizing the $S u b$ aru/HDS spectrum to the theoretical spectrum, using the previous fit and the model to determine the broad hydrogenBalmer and helium line profiles, but allowing the narrow-line absorption spectrum to be analyzed correctly. This is especially important for our analysis software which relies on a precise definition of the local continuum, and also for treating narrow lines which occur in the wings of much stronger lines. To confirm that the process works, the renormalized spectrum was analyzed for $T_{\mathrm{eff}}, \log g$ and $n_{\mathrm{He}}$ as before, giving results fully consistent with expectation.

\subsection{Line lists}

Having adopted a model atmosphere for the abundance analysis, the primary requirement for the abundance analysis is atomic data. Since we are making the assumption of LTE, the only data required for each line, are the wavelength, oscillator-strength, collisional and radiative damping constants (van der Waal's damping is included but not important), and the excitation potential of the level from which the line originates. In common with previous studies of intermediate helium hot subdwarfs (Naslim et al. 2011, 2013) this investigation encountered several lines rarely (if ever) seen in an astronomical context. Therefore considerable effort was made to check the completeness and reliability of the linelist adopted.

Construction of a line list to analyse blue-optical spectra $(\lambda 3600-5200 \AA)$ of early-type stars has progressed over several decades, commencing with LTE_LINES (Jeffery 1991), which focused on assessed data for single-line studies. This list was augmented to include other lines present in the spectra of mid- to late-B stars in order to synthesise large regions of the optical-blue spectrum (Woolf \& Jeffery 2002). Many of these data were contained within the compilation distributed by Kurucz \& Bell (1995). More recently, new atomic data were computed for zirconium, yttrium, germanium and lead lines discovered in helium-rich hot subdwarfs (Naslim et al. 2011, 2013). On computing a spectrum including all of these species to compare with the Subaru/HDS data, it was clear that many observed lines had no counterpart in the model, even after accounting for possible abundance excesses. Since UVO $0825+15$ is significantly hotter than most stars previously analyzed by us, on-line atomic databases were examined to assess whether other ions or more recent data could make up the deficit. The NIST Atomic Spectra Database (Kramida et al. 2015) was used extensively to identify possible candidates. Additional lines of $\mathrm{Ca}^{++}$and $\mathrm{S}^{++}$were identified in this way, and associated atomic were located in the Vienna Atomic Line Database (VALD) (Piskunov et al. 1995; Ryabchikova et al. 2015).

Unidentified lines. Efforts to identify additional lines included collating data for double and triply ionized species from VALD, setting artificially high abundances for these species and comparing the resultant spectra with observation. With over 150 lines still unidentified, other possibilities had to be considered:

1. Artefacts. Whilst instrumental artefacts or noise in the raw data can account for some very weak features, the vast majority are true absorption lines with equivalent widths above the detection threshold of $5 \mathrm{~m} \AA$.

2. Second Star. There is no evidence that the spectrum of a second star is present. Wherever a line was eventually identified it occurred at the correct wavelength (or radial velocity). Strong unidentified lines were checked against spectra of late-type stars, and chemically-peculiar A stars, with no matches found. Any such match would have been surprising since there is no evidence of a flux-excess at visual or infrared wavelengths from a cooler secondary.

3. Completeness. The line lists are almost certainly incomplete for stars of this effective temperature. It is likely that many arise from ions which are not represented (cf. Table A.1), but it is also possible that the weakest lines are due to absorption from high excitation states in ions already represented. 
Table 2. Elemental abundances for UVO $0825+15$ and related stars in the form $\log \epsilon_{i}=\log n_{i}+c$ (see text). Measurement errors are shown in parenthesis. The absence of a reported measurement is indicated by "-".

\begin{tabular}{|c|c|c|c|c|c|c|c|c|c|c|c|}
\hline Star & $\mathrm{H}$ & $\mathrm{He}$ & $\mathrm{C}$ & $\mathrm{N}$ & $\mathrm{O}$ & $\mathrm{Ne}$ & $\mathrm{Mg}$ & $\mathrm{Al}$ & $\mathrm{Si}$ & S & $\mathrm{Cl}$ \\
\hline 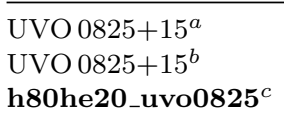 & $\begin{array}{l}11.8^{x} \\
11.8\end{array}$ & $\begin{array}{l}11.2^{x} \\
11.2\end{array}$ & $\begin{array}{l}<6.5 \\
{[6.5]} \\
6.0\end{array}$ & $\begin{array}{l}8.04(24) \\
8.07 \\
8.0\end{array}$ & $\begin{array}{l}7.43(07) \\
7.38 \\
7.2\end{array}$ & $\begin{array}{l}7.48(25) \\
7.85 \\
7.3\end{array}$ & $\begin{array}{l}6.25(11) \\
6.27 \\
7.0\end{array}$ & $\begin{array}{l}6.47(07) \\
6.26 \\
5.9\end{array}$ & $\begin{array}{l}6.26(21) \\
6.80 \\
6.9\end{array}$ & $\begin{array}{l}7.61(18) \\
7.71 \\
6.5\end{array}$ & $\begin{array}{l}6.34(11) \\
6.17 \\
{[5.0]}\end{array}$ \\
\hline $\begin{array}{l}\text { LS IV }-14^{\circ} 116^{1} \\
\text { HE } 1256-2738^{2} \\
\text { HE } 2359-2844^{2}\end{array}$ & $\begin{array}{l}11.83 \\
11.45 \\
11.58\end{array}$ & $\begin{array}{l}11.23(05) \\
11.44 \\
11.38\end{array}$ & $\begin{array}{l}8.04(22) \\
8.90(54) \\
8.51(29)\end{array}$ & $\begin{array}{l}8.02(20) \\
8.14(62) \\
8.00(57)\end{array}$ & $\begin{array}{l}7.60(17) \\
8.08(10) \\
7.81(16)\end{array}$ & $\begin{array}{l}<7.6 \\
<7.1 \\
<6.9\end{array}$ & $\begin{array}{l}6.85(10) \\
<6.5 \\
7.6(1)\end{array}$ & $\begin{array}{l}- \\
- \\
-\end{array}$ & $\begin{array}{l}6.32(12) \\
6.19(10) \\
5.73(13)\end{array}$ & $\begin{array}{l}- \\
<6.5 \\
<6.3\end{array}$ & $\begin{array}{l}- \\
- \\
-\end{array}$ \\
\hline $\begin{array}{l}\text { JL } 87^{3} \\
\text { PG 0909+276 } \\
\text { UVO } 0512-08^{4,5}\end{array}$ & $11.62(07)$ & $\begin{array}{l}11.26(18) \\
11.15(10) \\
11.23(10)\end{array}$ & $\begin{array}{l}8.83(04) \\
8.63(35) \\
8.59(20)\end{array}$ & $\begin{array}{l}8.77(23) \\
8.00(23) \\
7.94(21)\end{array}$ & $\begin{array}{l}8.60(23) \\
<7.50 \\
<7.75\end{array}$ & $\begin{array}{l}8.31(57) \\
<7.87 \\
-\end{array}$ & $\begin{array}{l}7.36(33) \\
- \\
-\end{array}$ & $\begin{array}{l}- \\
<6.25 \\
<6.25\end{array}$ & $\begin{array}{l}7.22(27) \\
5.80(10) \\
5.76\end{array}$ & $\begin{array}{l}6.88(1.42) \\
8.26(53) \\
8.14(49)\end{array}$ & $\begin{array}{l}- \\
- \\
-\end{array}$ \\
\hline 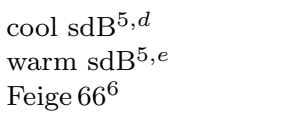 & & $\begin{array}{l}9.24(54) \\
10.15(76) \\
10.4\end{array}$ & $\begin{array}{l}6.99(47) \\
7.73(70) \\
6.79(30)\end{array}$ & $\begin{array}{l}7.68(41) \\
7.42(27) \\
7.65(15)\end{array}$ & $\begin{array}{l}7.88(26) \\
7.67(51) \\
-\end{array}$ & $\begin{array}{l}- \\
7.27(67) \\
-\end{array}$ & $\begin{array}{l}6.54(26) \\
7.17(29) \\
-\end{array}$ & $\begin{array}{l}5.70(18) \\
6.2 \\
<3.5\end{array}$ & $\begin{array}{l}6.79(37) \\
6.02(55) \\
<2.0\end{array}$ & $\begin{array}{l}6.51(21) \\
7.18(56) \\
7.69(46)\end{array}$ & $\begin{array}{l}- \\
- \\
-\end{array}$ \\
\hline $\operatorname{Sun}^{7}$ & 12.00 & [10.93] & 8.43 & 7.83 & 8.69 & [7.93] & 7.60 & 6.45 & 7.51 & 7.12 & 5.50 \\
\hline Star & $\mathrm{Ar}$ & $\mathrm{Ca}$ & $\mathrm{Ti}$ & $\mathrm{V}$ & $\mathrm{Fe}$ & $\mathrm{Ge}$ & $\mathrm{Sr}$ & $\mathrm{Y}$ & $\mathrm{Zr}$ & $\mathrm{Pb}$ & \\
\hline $\begin{array}{l}\text { UVO } 0825+15^{a} \\
\text { UVO } 0825+15^{b} \\
\text { h80he20_uvo0825 }\end{array}$ & $\begin{array}{l}<8.3 \\
{[6.6]} \\
5.9\end{array}$ & $\begin{array}{l}8.31(21) \\
8.14 \\
8.6\end{array}$ & $\begin{array}{l}7.37(34) \\
7.44 \\
{[7.6]}\end{array}$ & $\begin{array}{l}7.51(25) \\
7.46 \\
{[6.5]}\end{array}$ & $\begin{array}{l}<7.0 \\
7.30 \\
7.5\end{array}$ & $\begin{array}{l}6.24(06) \\
6.16 \\
{[3.7]}\end{array}$ & $\begin{array}{l}- \\
{[6.9]} \\
{[2.9]}\end{array}$ & $\begin{array}{l}5.37(09) \\
5.37 \\
{[2.2]}\end{array}$ & $\begin{array}{l}<5.3 \\
{[2.6]} \\
{[2.6]}\end{array}$ & $\begin{array}{l}5.49(18) \\
5.46 \\
{[1.9]}\end{array}$ & \\
\hline $\begin{array}{l}\text { LS IV }-14^{\circ} 116^{1} \\
\text { HE } 1256-2738^{2} \\
\text { HE } 2359-2844^{2}\end{array}$ & $\begin{array}{l}- \\
- \\
-\end{array}$ & $\begin{array}{l}- \\
- \\
-\end{array}$ & $\begin{array}{l}<6.0 \\
- \\
-\end{array}$ & $\begin{array}{l}<6.5 \\
- \\
-\end{array}$ & $\begin{array}{l}<6.8 \\
- \\
-\end{array}$ & $\begin{array}{l}6.28(12) \\
- \\
-\end{array}$ & $\begin{array}{l}6.96(15) \\
- \\
-\end{array}$ & $\begin{array}{l}6.16(10) \\
- \\
6.61(15)\end{array}$ & $\begin{array}{l}6.53(24) \\
- \\
6.47(15)\end{array}$ & $\begin{array}{l}- \\
6.39(23) \\
5.64(16)\end{array}$ & \\
\hline $\begin{array}{l}\text { JL } 87^{3} \\
\text { PG } 0909+276^{4,5} \\
\text { UVO } 0512-08^{4,5}\end{array}$ & $\begin{array}{l}- \\
8.68(15) \\
9.90\end{array}$ & $\begin{array}{l}- \\
7.81(35) \\
8.10(24)\end{array}$ & $\begin{array}{l}- \\
7.97(20) \\
8.06(33)\end{array}$ & $\begin{array}{l}- \\
8.10(26)^{y} \\
7.36(22)^{y}\end{array}$ & $\begin{array}{l}- \\
<7.87 \\
<7.81\end{array}$ & $\begin{array}{l}- \\
- \\
-\end{array}$ & $\begin{array}{l}- \\
- \\
-\end{array}$ & $\begin{array}{l}- \\
- \\
-\end{array}$ & $\begin{array}{l}- \\
- \\
-\end{array}$ & $\begin{array}{l}- \\
- \\
-\end{array}$ & \\
\hline $\begin{array}{l}\text { cool } \operatorname{sdB}^{5, d} \\
\text { warm } \operatorname{sdB}^{5, e} \\
\text { Feige } 66^{6}\end{array}$ & $\begin{array}{l}6.78(21) \\
7.89(17) \\
7.86(24)\end{array}$ & $\begin{array}{l}- \\
7.98(25) \\
8.09(20)\end{array}$ & $\begin{array}{l}6.30(35) \\
7.04(36) \\
6.96(22)\end{array}$ & $\begin{array}{l}7.10(36) \\
7.78(20) \\
6.37(22)\end{array}$ & $\begin{array}{l}7.58(20) \\
7.46(24) \\
6.46(17)\end{array}$ & $\begin{array}{l}- \\
- \\
5.21(05)\end{array}$ & $\begin{array}{l}- \\
- \\
-\end{array}$ & $\begin{array}{l}- \\
- \\
-\end{array}$ & $\begin{array}{l}- \\
- \\
-\end{array}$ & $\begin{array}{l}- \\
- \\
4.7\end{array}$ & \\
\hline $\operatorname{Sun}^{7}$ & {$[6.40]$} & 6.34 & 4.95 & 3.93 & 7.50 & 3.65 & 2.87 & 2.21 & 2.58 & 1.75 & \\
\hline
\end{tabular}

Notes:

$a$. abundances from line equivalent widths.

b. abundances from spectral synthesis $\chi^{2}$ minimization; values in square brackets were fixed (i.e. no solution was obtained).

c. model atmosphere input abundances as used in Fig. 2 and 3; values in square brackets refer to species for which continuous opacities were not included.

d. $25 \leq T_{\text {eff }} / \mathrm{kK} \leq 27$.

e. $35 \leq T_{\text {eff }} / \mathrm{kK} \leq 40$ excluding PG 0909+276 and UVO 0512-08.

$x$. from NOT/ALFOSC spectroscopy

$y$. from VIV.

References: 1. Naslim et al. (2011), 2. Naslim et al. (2013), 3. Ahmad et al. (2007), 4. Edelmann et al. (2003), 5. Geier (2013), 6.

O'Toole \& Heber (2006), 7. Asplund et al. (2009); photospheric except helium (helio-seismic), neon and argon (coronal).

\subsection{Methods}

The process of measuring abundances was carried out in two ways. The first approach was to compute a best-fit theoretical spectrum in which only elemental abundances were allowed to vary. A best-fit solution was obtained by $\chi^{2}$ minimization using a Levenburg-Marquardt method (Press et al. 1989), (SFIT: Jeffery et al. 1998) The result is shown in Appendix A.

A solution was sought for each element separately. Starting with an estimate of abundance (e.g. three times solar) a solution was sought and noted. This solution was then fixed in the starting mixture used for investigating subsequent elements. The final solution was obtained after iteration to allow for blended lines. Tests with different start estimates were made to insure against finding false local minima.
The second approach was to measure equivalent widths and compute abundances for selected lines from an individual curve-of-growth computed for each line. The microturbulent velocity was verified by the same means; the mean abundance obtained from $21 \mathrm{~N}$ II lines with $5<W_{\lambda} / \mathrm{m} \AA<$ 30 drops by less than 0.05 dex as $v_{\mathrm{t}}$ is increased from 2 to $20 \mathrm{~km} \mathrm{~s}^{-1}$; at this $T_{\text {eff }}$, the lines appear insensitive to small values of $v_{\mathrm{t}}$, probably because the latter are small relative to the thermal broadening at this effective temperature. We adopted $v_{\mathrm{t}}=2 \mathrm{~km} \mathrm{~s}^{-1}$ in line with Geier (2013). The lineby-line abundance measurements for unblended lines with atomic data available are shown in Appendix A.

The principal sources of error in the abundances arise from the atomic data, which need to be consistent across all ions and multiplets but which frequently are not (cf. S III, Appendix A: Fig. A.2), and from line misidentifications and 
blends. $\chi^{2}$-minimization uses all lines for which atomic data are available, whether present, blended or hidden in noise. The equivalent width approach uses primarily unblended lines with $W_{\lambda}>5 \mathrm{~m} \AA$. This systematic difference in the line samples, coupled with the error types identified, contributes to small differences provided by the two methods. Where significant, the equivalent-width measurements are preferred.

From the width of the lead absorption lines, the projected rotation velocity is small. The overall quality of the spectrum prevents us from setting a limit any stronger than $v \sin i<10 \mathrm{~km} \mathrm{~s}^{-1}$

\subsection{Abundances}

Abundances derived for each element identified in the spectrum of UVO $0825+15$ are shown in Table 2, together with those adopted for the model atmosphere and data for comparable stars. Abundances are given in the form $\log \epsilon_{i}=$ $\log n_{i}+c$ where $\log \Sigma_{i} a_{i} \epsilon_{i}=12.15, a_{i}$ are atomic weights, and $c=\log \Sigma \epsilon_{i}$. This conserves values of $\epsilon_{i}$ for elements whose abundances do not change, even when the mean atomic mass of a mixture changes substantially, and conforms to the convention that $\log \epsilon_{\mathrm{H}} \equiv 12$ for the Sun and other hydrogen-normal stars.

Errors are based on the standard deviation of the line abundances about the mean or, in the case of a single representative line, on the estimated error $( \pm 2 \mathrm{~m} \AA)$ in the equivalent width measurement (Table A.1), and an assumed error of $\pm 10 \%$ in the transition probability. In the case of UVO $0825+15$ upper limits were obtained by computing the abundance required to give a line with an equivalent width of $5 \mathrm{~m} \AA$, the detection threshold in the Subaru/HDS spectrum.

Carbon is not detected in the spectrum of UVO $0825+15$. The strongest predicted line CiII $\lambda 4070.3 \AA$ is not present. With abundance $\log \epsilon_{\mathrm{C}}=6.5$ (2 dex below solar) this line would be predicted to have an equivalent width $5.6 \mathrm{~mA}$. Similarly, iron is at the boundary of detectability, although a solution is obtained with SFIT. Notably, germanium, yttrium and lead are measured with abundances $2.5-4$ dex above solar.

A few discrepancies between the equivalent-width, $\chi^{2}$ minimization and input model abundances persist (Table 2). The model inputs are restricted, with elements heavier than oxygen being defined in groups rather than individually and their abundances being chosen as a compromise amongst several elements. Reasons for differences between line-byline and $\chi^{2}$ measurements have been discussed above.

Heavy-metal subdwarfs. For comparison with UVO $0825+15$ abundances for the zirconium-rich LS IV $-14^{\circ} 116$ and two intermediate He-sds with extreme overabundances of lead, HE 2359-2844 and HE 1256-2738, are shown in Table 2 .

Intermediate-helium subdwarfs. Apart from hydrogen and helium, the prototype intermediate helium subdwarf JL 87 appears to have a roughly solar-like surface composition Ahmad et al. (2007), but no elements heavier than sulphur were measured. Edelmann et al. (2003) identified two intermediate helium-rich subdwarfs, PG 0909+276
Table 3. Principal frequencies and semi-amplitudes ( $p p t=$ parts per thousand) measured from the entire $K 2$ dataset and for subsets of the K2 data for UVO $0825+15$.

\begin{tabular}{rrrrrrrr}
\hline$K_{2} t_{0^{-}} t_{1}$ & $\begin{array}{r}\delta f \\
\mathrm{~d}\end{array}$ & $\begin{array}{r}f_{1} \\
\mathrm{~d}^{-1}\end{array}$ & $\begin{array}{r}\sim 2 f_{1} \\
\mathrm{~d}^{-1}\end{array}$ & $\begin{array}{r}f_{2} \\
\mathrm{~d}^{-1}\end{array}$ & $\begin{array}{r}a_{f 1} \\
\mathrm{ppt}\end{array}$ & $\begin{array}{r}a_{2 f 1} \\
\mathrm{ppt}\end{array}$ & $\begin{array}{r}a_{2} \\
\mathrm{ppt}\end{array}$ \\
\hline $2306.4-81.4$ & 0.013 & 2.237 & 4.497 & 1.861 & 1.19 & 0.12 & 0.40 \\
$2306.6-14.1$ & 0.133 & 2.077 & 4.126 & & 2.36 & 0.92 & \\
$2314.1-21.6$ & 0.133 & 2.278 & 3.930 & 1.822 & 2.52 & 0.26 & 1.36 \\
$2321.6-29.0$ & 0.133 & 2.248 & 4.477 & 1.828 & 3.36 & 0.37 & 1.77 \\
$2329.0-36.5$ & 0.135 & 2.252 & 4.494 & 1.778 & 3.40 & 0.43 & 1.30 \\
$2336.5-44.0$ & 0.133 & 2.228 & 4.448 & 1.905 & 3.42 & 0.42 & 0.62 \\
$2344.0-51.5$ & 0.133 & 2.200 & 4.391 & 1.860 & 3.22 & 0.40 & 0.65 \\
$2351.5-59.0$ & 0.133 & 2.197 & 4.407 & & 2.17 & 0.21 & \\
$2359.0-66.5$ & 0.133 & 2.054 & 3.919 & & 0.40 & 0.08 & \\
$2366.5-74.0$ & 0.133 & 1.909 & 3.824 & 1.909 & 0.35 & 0.05 & 0.35 \\
$2374.0-81.4$ & 0.133 & 2.073 & 4.257 & & 0.67 & 0.05 & \\
$2306.6-66.6$ & 0.017 & 2.236 & 4.497 & 1.858 & 1.47 & 0.15 & 0.52 \\
\hline
\end{tabular}

and UVO 0512-08, with extreme overabundances of some iron-group elements, including scandium, titanium, vanadium, manganese and nickel, but not iron (Table 2). Eight other intermediate helium subdwarfs for which abundances have been measured show no detectable excesses (Naslim et al. 2010, 2012, 2013) although, in many cases, even substantial excesses could not be detected with available spectra. Abundance analyses for other intermediate helium stars having $0.1<n_{\mathrm{He}}<0.9$ and no measured excess in any of $\mathrm{Ge}, \mathrm{Sr}, \mathrm{Y}, \mathrm{Zr}$, or $\mathrm{Pb}$ include BPS CS 22956-0094 (Naslim et al. 2010), CPD-20¹123 (Naslim et al. 2012), HE 0111-1526, HE 1135-1134, HE 1238-1745, HE 1258+0113, HE 1310-2733, and HE 2218-2026 (Naslim et al. 2013).

Normal subdwarfs. Geier (2013) published surface abundances for a sample of 106 hot subdwarfs, the large majority of which are helium poor. He demonstrated that some elements show significant trends with effective temperature. Table 2 includes mean abundances in two ranges of $T_{\text {eff }}$, representing cool and warm sdB stars, between which there is a smooth trend across the entire sdB temperature range (Fig. 8). The warm group corresponds to the temperature range of the heavy-metal subdwarfs. Both extremes demonstrate significantly sub-solar abundances of light elements, notably helium, oxygen, magnesium, aluminium, and silicon, roughly solar abundances of nitrogen and iron, and super-solar abundances of calcium, titanium and vanadium. Carbon is under-abundant at effective temperatures below $35 \mathrm{kK}$, above which the mean value approaches solar. O'Toole \& Heber (2006) were able to explore additional elements using HST spectroscopy of five hot subdwarfs, establishing, further to the above, super-solar abundances of lead and, in some cases, germanium. Of these, Table 2 includes the results for Feige 66 .

\section{LIGHT CURVE ANALYSIS}

A first examination of the light curve is confusing. The first part shown in Fig. 1 suggests a period around $0.5 \mathrm{~d}$ modulated by a signal having a beat period of about 5 days. The 


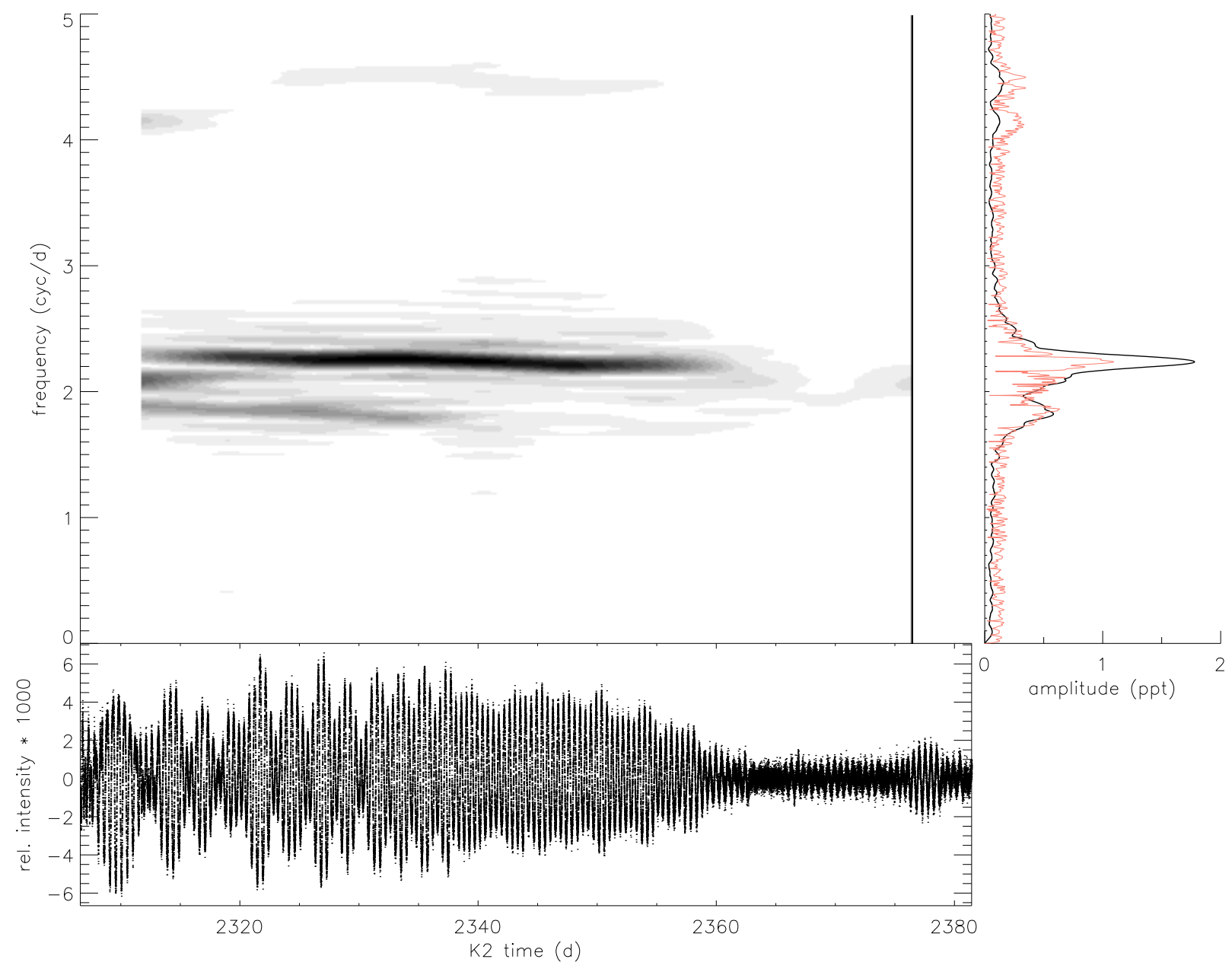

Figure 5. Main panel: the sliding amplitude spectrum of the K2 light curve of UVO $0825+15$ shown as greyscale, based on data blocks of duration $\Delta T=10 \mathrm{~d}$ sampled approximately every $3.7 \mathrm{~d}$. The frequency resolution $(1 / \Delta T)$ is hence $\pm 0.1 \mathrm{~d}^{-1}$. The light curve is reproduced at the same horizontal scale in the panel beneath. The panel on the right shows, in bold, the time-averaged amplitude spectrum, and in light red, the amplitude spectrum of the entire dataset. This figure demonstrates the presence of power around two principal frequencies at $2.23 \pm 0.01$ and $1.82 \pm 0.04 \mathrm{~d}^{-1}$ and at higher frequencies.

$0.5 \mathrm{~d}$ period appears to be present throughout most of the K2 observations (Fig. 1), with significant amplitude modulation at early times, little modulation at middle times, and decaying to negligible amplitude at late times. In order to interpret the light curve, it is first necessary to establish its period content.

An inspection of the amplitude spectrum obtained from the discrete Fourier transform is equally perplexing. The amplitude spectrum for the entire K2 light curve shows a group of peaks with frequencies $f$ around $2 \mathrm{~d}^{-1}$, a significant peak at $f_{1} \approx 2.2 \mathrm{~d}^{-1}$, and some power at $\approx 2 f_{1}$. The amplitude of the signal at $f_{1}$ is, however, substantially weaker than that seen in a large part of the light curve. Dividing the light curve into segments shows that there is considerable variation in the amplitude spectrum over time (Fig1). Two primary reasons suggest themselves: i) the signal is nonuniform, varying in both amplitude and frequency and ii) there are multiple signals present and unresolved in the rel- atively short duration of the K2 campaign. Other interpretations are also be possible.

The light curve was investigated using a sliding Fourier transform, an example of which is shown in Fig. 5. The choice of the duration of dataset in each element of the sliding transform is a compromise between temporal and frequency resolution; samples of duration $10 \mathrm{~d}$ giving a frequency resolution of $\approx 0.1 \mathrm{~d}^{-1}$ were found to give the most coherent picture of the current data. The light curve was also investigated by dividing into ten discrete subsamples, computing the amplitude spectrum, and measuring the frequency and amplitude of the principal peaks for each sample, as well as for the entire spectrum and various other subsamples (Table 3).

The picture that emerges from this analysis is that a dominant oscillation with $f_{1} \approx 2.24 \pm 0.01 \mathrm{~d}^{-1}$ and semiamplitude $a_{1} \approx 3$ parts per thousand (ppt) persists throughout most of the K2 light curve. At early times, an addi- 
tional oscillation with $f_{2} \approx 1.8 \mathrm{~d}^{-1}$ is observed. The relative amplitude of the two signals is not well determined; typically $a_{1} / a_{2} \approx 2-5$. In Fig.5, $f_{1}$ appears to vary, but only by an amount which is less than the frequency resolution $\left( \pm 0.1 \mathrm{~d}^{-1}\right)$. At the very start of the $K 2$ campaign $f_{1}$ appears to be significantly shorter at $\approx 2.08 \pm 0.13 \mathrm{~d}^{-1}$. Such behaviour might be anticipated if, for example, the frequency content of the power spectrum is not fully resolved. The frequency resolution of the entire dataset is $\delta f \approx 0.013 \mathrm{~d}^{-1}$. Signals which can only be identified for a part of the time series are less well resolved $\left(\delta f \approx 0.13 \mathrm{~d}^{-1}\right)$. For signals with frequencies $\approx 2 \mathrm{~d}^{-1}$, both resolutions are low; the data thus suggest the presence of multiple unresolved closely-spaced frequencies.

On the basis of available evidence, it appears that the light variations in UVO $0825+15$ can be interpreted in terms of a multi-periodic signal with multiple unresolved frequencies around 1.8 and $2.2 \mathrm{~d}^{-1}$ (periods 10.8 and $13.2 \mathrm{~h}$ ). The usual explanation for such a signal is the presence of global oscillations occurring simultaneously in a number of modes. For a star of the dimensions of UVO $0825+15$, the fundamental radial mode would have a period of $\approx 300 \mathrm{~s}$ (Jeffery \& Saio 2016); if the oscillations arise from UVO $0825+15$, they must be associated with gravity modes of extremely high radial order. There is currently no known mechanism which could excite such modes in UVO $0825+15$, which is an even more extreme example than LS IV $-14^{\circ} 116$ in which gmodes with periods of $\approx 1800 \mathrm{~s}$ have been observed (Ahmad \& Jeffery 2005; Green et al. 2011).

Other interpretations for light variations with the observed period and amplitude must be considered. Reflection from a low-mass companion (a planet perhaps) with an inhomogeneous surface might suffice, especially if rotating asynchronously. However, assuming an albedo of unity, a Jupiter mass planet in a 12 hour orbit would reflect $\approx 0.6$ ppt of the parent star's light. A $0.3 \mathrm{M}_{\odot}$ dwarf could reflect $\approx 4 \mathrm{ppt}$ of the parent starlight, but would require an orbital velocity $\approx 90 \mathrm{~km} \mathrm{~s}^{-1}$ from the primary. Reducing the inclination to match the non-detection of orbital motion ( $(2.2)$ would reduce the apparent light variation below that detected. Companions of sufficiently low mass $\left(\leq 0.01 \mathrm{M}_{\odot}\right)$ and large radius $\left(\geq 0.25 \mathrm{R}_{\odot}\right)$ to satisfy the radial velocity and light curve constraints are otherwise difficult to identify in astronomical terms. Further difficulties with such a solution include the apparent drift in frequency, and the absence of evidence of any infrared excess $(\$ 3.2)$. Geometrical effects due to tidal distortion by a massive companion can be ruled out on similar grounds. The possibility of light modulation by magnetically supported surface spots was discussed in the context of LS IV $-14^{\circ} 116$ by Ahmad \& Jeffery (2005), discounted by Green et al. (2011), and eliminated by Randall et al. (2015). However, it remains necessary to consider a differentially rotating surface with migrating spots as a possible light curve driver. Combining the upper limit to $v_{\text {rot }} \sin i<10 \mathrm{~km} \mathrm{~s}^{-1}$ $(\S 4)$ with a typical hot subdwarf radius $\approx 0.12 \mathrm{R}_{\odot}$ gives a miniumum rotation period of $\approx 14.6 \mathrm{~h}$, which is almost compatible with the observed periods of 11-13 h.

Due to the large size of the detector pixels $\left(\approx 4^{2}\right.$ arcsec), a problem frequently encountered in the analysis of both Kepler and K2 data is contamination by light variation of a nearby star (cf. Silvotti et al. 2014) or, less frequently, charge leakage from a bright star occupying the same CCD
Table 4. Kinematical properties for UVO $0825+15$.

$\begin{array}{ll}\text { distance } & d=257_{-29}^{+37} \mathrm{pc} \\ \text { space motion } & U=-14 \pm 2 \mathrm{~km} \mathrm{~s}^{-1} \\ & V=+303 \pm 18 \mathrm{~km} \mathrm{~s}^{-1} \\ & W=-32 \pm 2 \mathrm{~km} \mathrm{~s}^{-1} \\ \text { apocenter radius } & R_{\mathrm{a}}=14.3 \pm 0.4 \mathrm{kpc} \\ \text { eccentricity } & e=0.26 \pm 0.01 \\ \text { galactic rest frame velocity } & v_{\mathrm{grf}}=+298 \pm 14 \mathrm{~km} \mathrm{~s}^{-1} \\ z \text {-component of angular mom } & J_{z}=2502 \pm 23 \mathrm{kpc} \mathrm{km} \mathrm{s}^{-1} \\ \text { maximum height above plane } & z_{\max }=0.80 \pm 0.04 \mathrm{kpc}^{\mathrm{max}}\end{array}$

column. The 2MASS catalogue shows 5 stars within 1 arcmin of UVO $0825+15$, all having $m_{\mathrm{J}}>15.3$, the closest having $m_{\mathrm{J}}=16.3$ is 20 arcsec distant (UVO 0825+15 has $\left.m_{\mathrm{J}}=12.4\right)$. None are bright enough to contaminate the target in the manner observed. We found no evidence for any other potential source of contamination despite investigating i) the behaviour of individal sky pixels in the mask, ii) the light curves for all stars brighter than $14^{\text {th }}$ mag. within 10 arcmin, and iii) potential contaminants in the full-frame image for UVO $0825+15$. Furtermore, there is no evidence for an unresolved companion in the flux distribution (§3.2).

The resemblance of the UVO $0825+15$ light curve to those of slowly pulsating B stars, especially KIC 11293898 (cf. McNamara et al. 2012), is remarkable and suggestive. From inspection of the complete light curve obtained over the entire Kepler mission, KIC 11293898 shows an amplitude similar to that of UVO $0825+15$, with a rich cluster of well-resolved g-modes at frequencies around $2.4 \mathrm{~d}^{-1}$, with harmonics at twice this value (and beyond). UVO $0825+15$ might be expected to show similar behaviour if observed continuously for 3 years. How this can be reconciled with its spectroscopic properties remains a mystery.

\section{KINEMATICS}

Evidence for peculiar galactic orbits amongst other intermediate helium- and heavy-metal-rich subdwarfs (Randall et al. 2015; Martin et al. 2016) and measurement of a large proper motion in UVO $0825+15$ ( $\mathrm{H} \varnothing \mathrm{g}$ et al. 2000) raises the question of kinematics and the identity of the star's parent population.

Using the measured value for surface gravity and an assumed mass typical for other subdwarfs $\left(0.50 \pm 0.1 \mathrm{M}_{\odot}\right)$ leads to a stellar radius of $r=0.121_{-0.014}^{+0.018} \mathrm{R}_{\odot}$. The angular diameter then yields a distance $d=257_{-29}^{+37} \mathrm{pc}$. Together with published proper motion measurements and the measurement of radial velocity from the Subaru/HDS spectrum, the galactic orbit can be computed (Martin et al. 2016). The components of space motion and Galactic orbital elements are given in Table 4.

With a Galactic rotation velocity of $V=+303 \mathrm{~km} \mathrm{~s}^{-1}$, UVO $0825+15$ is rotating slightly faster than the Local Standard of Rest $\left(242 \mathrm{~km} \mathrm{~s}^{-1}\right)$ and is typical of the thick disk population. Fig. 6 shows the orbit morphology. The orbit extends $0.8 \mathrm{kpc}$ above the plane and has an orbital eccentricity of 0.26 , also indicative of thick disk membership. 

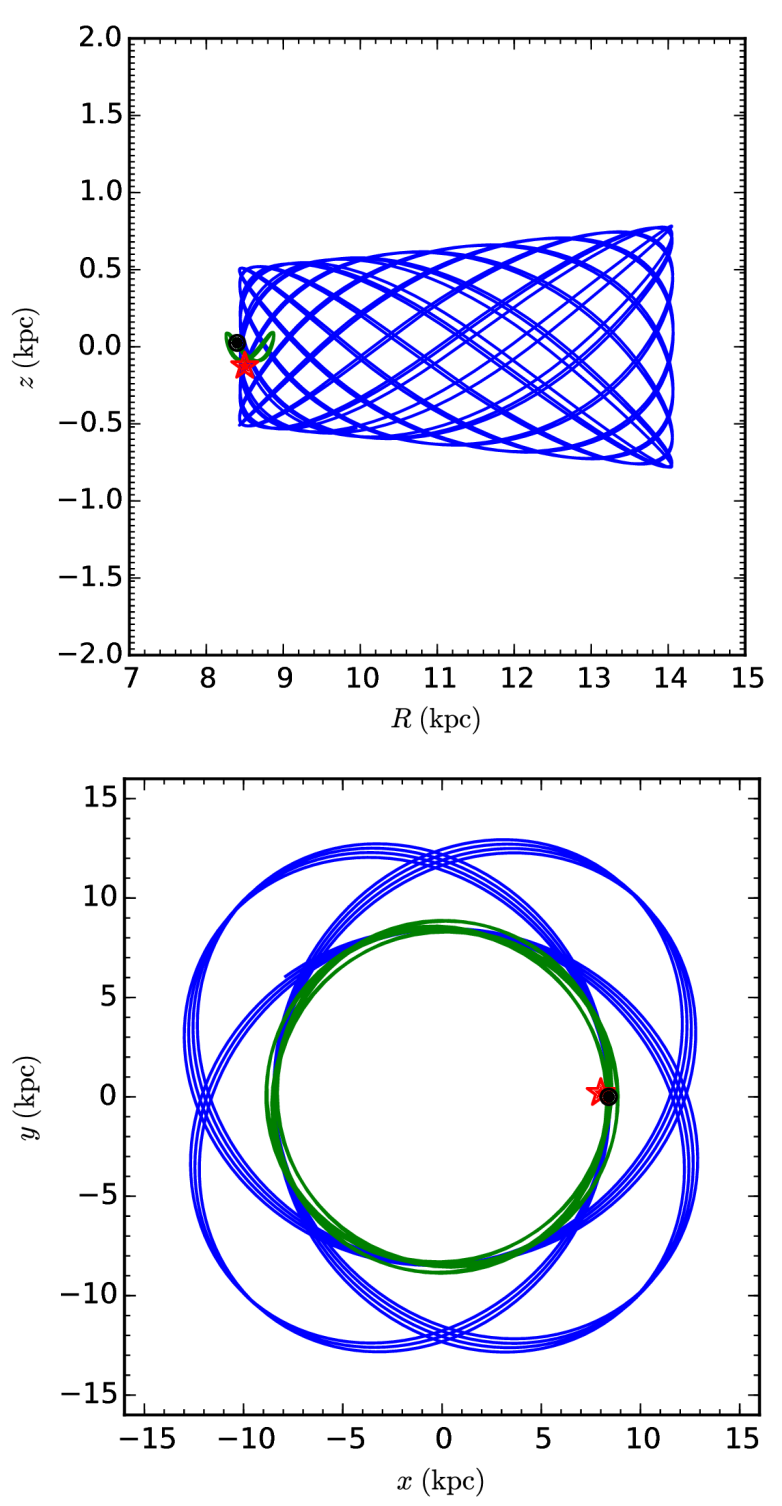

Figure 6. The current position (red star) and galactic orbit (blue) of UVO $0825+15$ projected forward over 3 Gyr. Top: meridional section $(R-z)$. Bottom: projection onto the Galactic plane $(x-y)$. The current position (black circle) and projected orbit (green) of the Sun are also shown.

\section{UVO 0825+15 AND OTHER HOT SUBDWARFS}

Figures 7 and 8 place UVO $0825+15$ in context with other hot subdwarfs, including the "normal" helium-deficient subdwarf B and subdwarf O stars, helium-rich hot subdwarfs, and a selection of chemically-peculiar intermediate heliumrich hot subdwarfs. The latter, which include UVO $0825+15$, lie in the range $30000<T_{\text {eff }} / \mathrm{K}<40000$ and on or slightly above the helium main sequence. They are sufficiently hot that they cannot be "extended horizontal-branch" stars which retain a remnant hydrogen-rich envelope. Their position on the helium main-sequence does suggest, but does not require, that they have helium-burning cores. If so, whatever hydrogen remains in their surface is sufficiently reduced in deeper layers that its opacity plays no role in the overall structure of the star. JL 87 appears to represent another type of intermediate helium-rich subdwarf, being cooler and having a near-solar distribution of light elements (Ahmad et al. 2007).

\subsection{Chemistry}

The group including UVO $0825+15$, LS IV $-14^{\circ} 116$, HE 2359-2844, and HE 1256-2738 represent the true "heavy-metal" stars, having 3-4 dex excesses of some of germanium, strontium, yttrium, zirconium, and lead, this being at least 1 dex higher than seen in any other hot subdwarf. UVO 0512-08 and PG 0909+276 show very high excesses of iron-group elements, but no detection of the heavy metals. The simplest interpretation of the peculiar chemistries of all six stars is that their radiation-dominated photospheres are modified by diffusive processes, including gravitational settling of heavy species and radiative levitation. The latter will concentrate atomic species into layers where their specific opacities are high, generally corresponding to the ionization temperatures of specific ions. If these layers also correspond to layers in the photosphere where absorption lines form, then they will be observed with large overabundances. The converse will also be true; ions of low specific opacity in the line-forming layer will migrate upwards or downwards and hence appear under-abundant.

Significantly, none of these groups, including the normal sdB stars shows any surface excess of iron; it may be depleted in some cases. As well as its heavy-metal overabundances, UVO $0825+15$ is remarkable, and possibly unique, for its very low surface carbon abundance. It is tempting to use the iron abundance as an indicator of overall metallicity, and the carbon/nitrogen/oxygen ratio as evidence that the hydrogen-depleted surface is the product of CNO-process hydrogen-burning. This is almost certainly misleading, since it directly contradicts the argument that diffusion is responsible for the exotic chemistry of heavy elements. Similarly, other explanations, including contamination by a supernova explosion, or the dredge-up of s-process elements in an asymptotic giant branch star fail primarily because of the low light-element abundances (especially carbon) and the normal iron abundance.

\subsection{Variability}

The UVO $0825+15$ light curve was discussed in $\S 5$; most common explanations for the amplitude and frequencies were deemed not viable. An explanation in terms of nonradial g-mode oscillations is favoured on the grounds that more than one frequency is present, and the amplitude is variable; the latter could be explained in terms of beating between unresolved modes. However, pulsation is not favoured on the grounds that the non-radial orders would have to be very high in order to account for the periods, which are very long for hot subdwarfs. In particular they lie well beyond the theoretical g-mode cutoff boundary as articulated for white dwarfs by Hansen et al. (1985) and which corresponds to $P_{g} L \leq 3600 \mathrm{~s}$ in the current case, where $L^{2}=\ell(\ell+1)$. The absence of a known driving mechanism is also a factor, but with radiative levitation in operation at the surface, an undi- 


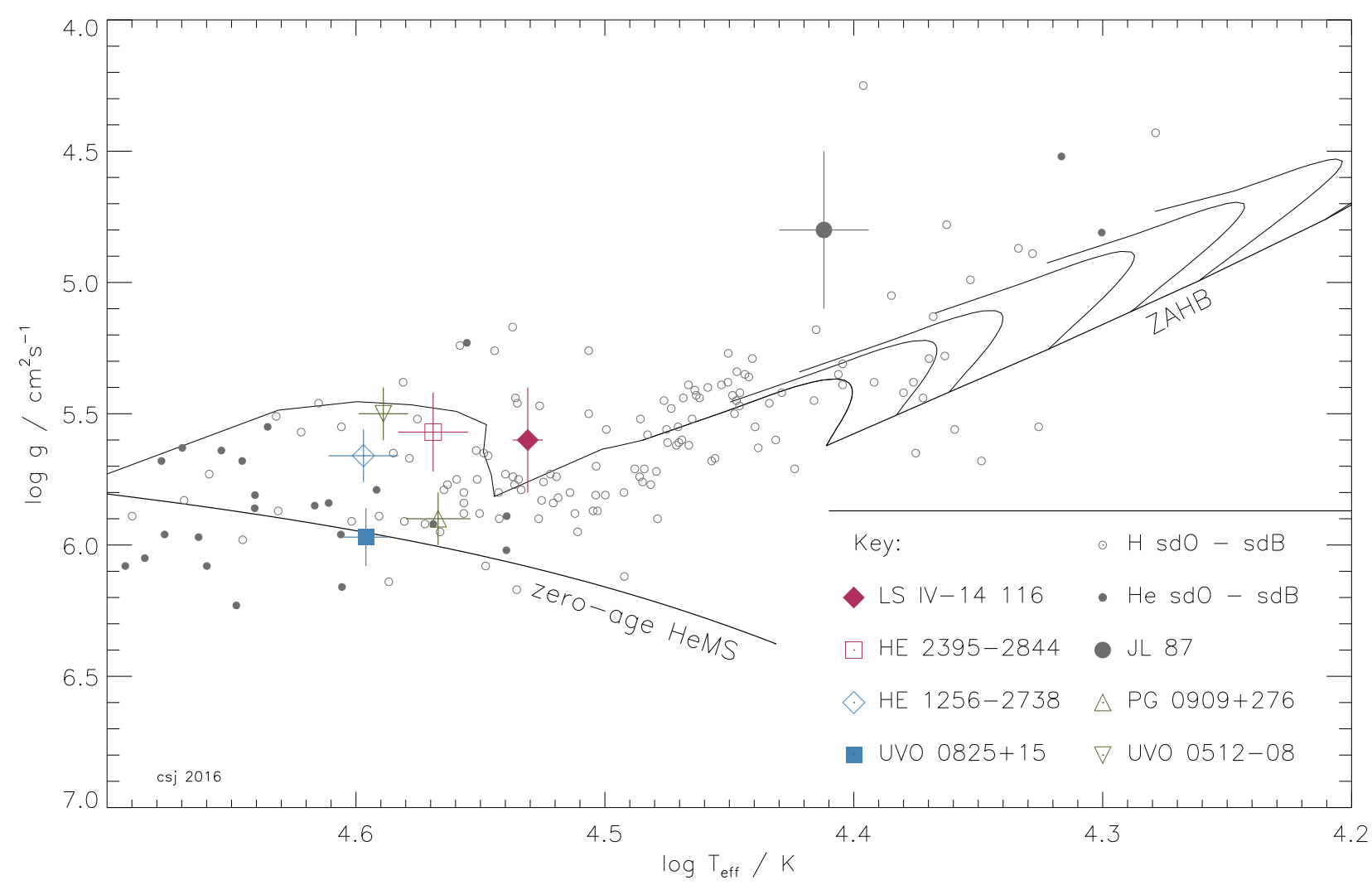

Figure 7. The distribution of chemically-peculiar, helium-rich and normal hot subdwarfs with effective temperature and surface gravity. Solid lines show representative positions for the theoretical zero-age helium main-sequence (HeMS: $Z=0.02)$ and models evolving from the zero-age horizontal branch (ZAHB) to the end of core helium burning (Dorman et al. 1993, ; z22: $M_{\mathrm{c}}=0.469, Y=0.288,[\mathrm{Fe} / \mathrm{H}]=0.0$ ). The post-HB evolution of one model $\left(M=0.471 \mathrm{M}_{\odot}\right)$ is also shown. The observed data are from this paper, Edelmann et al. (2003); Ahmad et al. (2007); Naslim et al. (2011, 2013) and Németh et al. (2012).

agnosed opacity bump in the interior would be a likely possibility. Indeed, the excitation of g-modes in Wolf-Rayet stars has been attributed to a similar phenomenon (Townsend \& MacDonald 2006). In order to place the UVO 0825+15 light curve into a wider context, it is worth comparing it with other variable and peculiar hot subdwarfs.

LS IV $-14^{\circ} 116$. Pulsations in chemically-peculiar heliumrich subdwarfs were first discovered by Ahmad \& Jeffery (2005), who discovered light variations on timescales of $\approx 1800 \mathrm{~s}$ in the intermediate helium-rich hot subdwarf LS IV-14 $116\left(T_{\text {eff }}=32500 \mathrm{~K}, \log \mathrm{g} / \mathrm{cm} \mathrm{s}^{-2}=5.4\right.$, $\log n_{\mathrm{He}} / n_{\mathrm{H}}=-0.58$, (Ahmad \& Jeffery 2003)). Fine analysis showed the surface of LSIV $-14^{\circ} 116$ to have $\sim 4$ dex overabundances of zirconium, strontium, yttrium and germanium, which as so far been attributed to the effects of selective radiative levitation in the stellar photosphere (Naslim et al. 2011). Whilst the light variations have subsequently been confirmed and interpreted as non-radial g-mode pulsations (Jeffery 2011; Green et al. 2011), there is debate over the precise effective temperature (Naslim et al. 2011; Green et al. 2011), and no completely satisfactory driving mechanism has been identified. An argument that the $\epsilon$-mechanism is active remains to be tested (Miller Bertolami et al. 2011). - need to refer to green $+11 \log \mathrm{g}$ as well ... might be woirth looking at $P g$ for this case also.-
$\mathrm{KIC} \mathbf{1 7 1 8 2 9 0}=(\mathrm{SDSS}) \mathbf{J 1 9 2 3 0 0 + 3 7 1 5 0 4}$ is a blue horizontal-branch star or 'cool' hot subdwarf ( $T_{\text {eff }}=$ $\left.22100 \mathrm{~K}, \log g / \mathrm{cm} \mathrm{s}^{-2}=4.72\right)$ with a super-solar helium abundance $\left(\log n_{\mathrm{He}} / n_{\mathrm{H}}=0.45\right)$ (Østensen et al. 2012). It was observed with Kepler as an exoplanet target, revealing a rich spectrum of low-amplitude pulsation modes with periods between one and twelve hours, most of which follow a regular spacing of $276.3 \mathrm{~s}$. These fall into the classical range for g-mode non-radial pulsations (Jeffery \& Saio 2006). Although having a $12 \mathrm{~h}$ period and super-solar helium abundance in common, the large difference in $T_{\text {eff }}$ means that this star provides negligible insight for the present study.

$\mathrm{KIC} 10449976=(2 \mathrm{MASS}) \mathrm{J} 184714+474146$ is an extremely helium-rich subdwarf $\left(T_{\text {eff }}=40000 \mathrm{~K}\right.$, $\log g / \mathrm{cm} \mathrm{s}^{-2}=5.3$ and $\left.\log n_{\mathrm{He}} / n_{\mathrm{H}}=+1.2\right)$ (Jeffery et al. 2013). Kepler photometry (quarters 3 and 5-9) shows evidence for a periodic modulation on a time-scale of $\approx 3.9 \mathrm{~d}$, but with variable amplitude. Radial-velocity measurements over a $5 \mathrm{~d}$ time-scale show an upper variability limit of $\approx 50 \pm 20 \mathrm{~km} \mathrm{~s}^{-1}$. The origin of this modulation remains to be confirmed; Bear \& Soker (2014) argue for reflection from a weather-affected planet but fail to show how such a model can lose phase coherence over an interval of 160 or more orbits. Follow-up observations are yet to be carried out. 


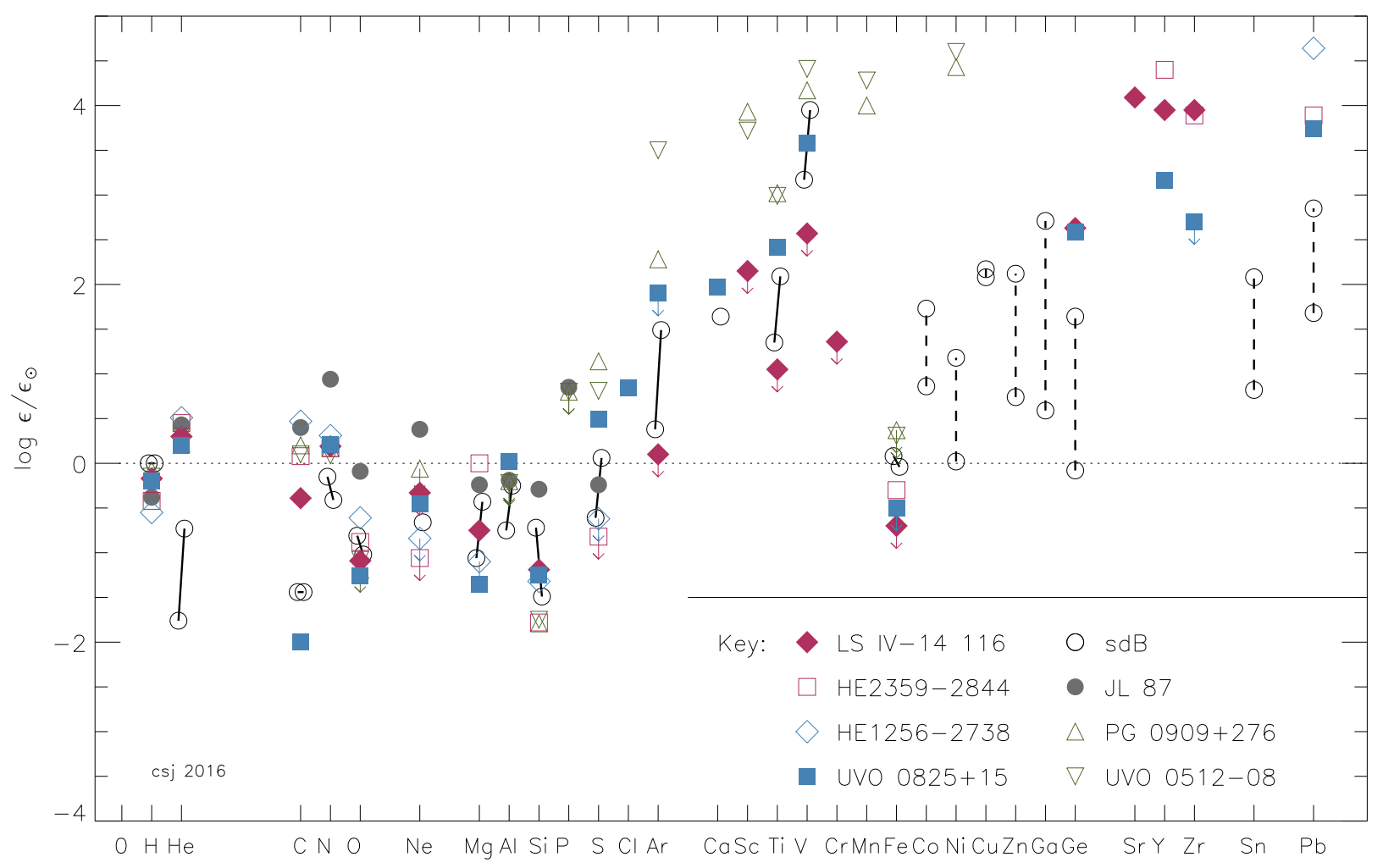

Figure 8. Surface abundances of super metal-rich hot subdwarfs, including the pulsating stars LS IV $-14^{\circ} 116$ and UVO $0825+15$ (Edelmann et al. 2003; Naslim et al. 2011, 2013) and this paper. Abundances are shown relative to solar values (Asplund et al. 2009, dotted line: ). Mean abundances and ranges for the helium-rich subdwarf JL 87 (Ahmad et al. 2007) and for normal subdwarfs are also shown. The latter are shown by connected open circles as (i) $Z \leq 26$ (solid lines): the abundance distribution across the temperature range for sdBs as defined in Table 2, with cool and warm groups displaced left and right respectively (Geier 2013) and (ii) $Z \geq 27$ (broken lines): the range of abundances measured for five normal sdBs from ultraviolet spectroscopy (O'Toole \& Heber 2006).

KIC $9408967=($ SDSS $) \mathbf{J 1 9 3 5 2 + 4 5 5 5 . ~ Ø s t e n s e n ~ e t ~ a l . ~}$ (2010) identify three He-sdOB stars in the Kepler field: (Galex) J19034+3841, (SDSS) J19352+4555 and J19380+4649. None pulsate, but Østensen et al. (2010) reported rapid drops in the Kepler Quarter 2 photometry of J19352+4555 with no apparent regularity. The maximum light variations in the other two He-sdOBs at low frequencies $(100-500 \mu \mathrm{Hz})$ are 117 parts per million $(\mathrm{J} 19034+3481)$ and 29 parts per million (J19380+4649). J19352+4555 is reported to be an extremely He-rich sdO star with $\left(T_{\text {eff }}, \log g\right)$ similar to that of UVO $0825+15$ (Østensen 2016, private communication). Reprocessed Kepler light curves of J19352+4555 from Quarters 2 (short cadence and long cadence) and 10 (long cadence only) were extracted from the Kepler archive. Examination of these shows no evidence for periodic short-term variability with an amplitude greater than 60 parts per million $(2.5 \sigma)$.

V499 Ser $=($ SDSS $) \quad \mathbf{J 1 6 0 0 4 3 . 6 + 0 7 4 8 0 2 . 9}$ was found by Woudt et al. (2006) to be a very rapid hot sdO pulsator, with pulsation periods in the range $60-120 \mathrm{~s}$. With $T_{\text {eff }}=68500 \pm 1770 \mathrm{~K}, \log g / \mathrm{cm} \mathrm{s}^{-2}=6.09 \pm 0.07$, and $\log n_{\mathrm{He}} / n_{\mathrm{H}}=-0.64 \pm 0.05$ (Latour et al. 2011), it is much hotter than UVO $0825+15$, but the oscillation periods are compatible with being p-modes. Fontaine et al. (2008) ar- gue that, as for the cooler sdB pulsators, radiative levitation can accumulate sufficient iron and other high-opacity species to drive low-order low-degree p-mode pulsations in some hot subdwarfs with $T_{\text {eff }}$ between 60000 and $80000 \mathrm{~K}$ by the $\kappa$-mechanism. Again, the surface abundance of helium is super-solar and similar to that in UVO $0825+15$.

EO Cet = PB 8783 was originally identified as a classical sdB pulsator (Koen et al. 1997) having periods in the range $120-135 \mathrm{~s}$ and an early F-type companion. Subsequent spectroscopy showed HeII $\lambda 4686 \AA$ to be present, indicating $T_{\text {eff }}$ well in excess of $36000 \mathrm{~K}$; this star may consequently have been the first pulsating sdO star discovered (Østensen 2012). With similarities to V499 Ser, further spectroscopy is required.

$\omega$ Cen has been found to show a pulsation instability strip containing five hot subdwarfs with multi-periodic oscillations with periods between 85 and $125 \mathrm{~s}$ and with $T_{\text {eff }}$ between 48000 and $54000 \mathrm{~K}$ (Randall et al. 2016). All are helium-poor. These p-mode pulsations are consistent with theoretical predictions and a consequence of radiativelydriven diffusion in the interior enhancing the the iron-bump opacities, just as in the case of V499 Ser. Six short-period $(85-150 \mathrm{~s})$ pulsating hot subdwarfs have been detected in 
the globular cluster NGC 2808 (Brown et al. 2013), but are not strongly constrained in $T_{\text {eff }}$.

Thus there is evidence of both p- and g-mode pulsations in helium-poor and helium-rich hot subdwarfs with $T_{\text {eff }}>$ $30000 \mathrm{~K}$. There is evidence of irregular and longer-term (3.9 d) light variations in at least one case (KIC 10449976), which has yet to be explained. One blue horizontal-branch star (KIC 1718290) shows g-mode pulsation periods of up to $12 \mathrm{~h}$; these can be reconciled with pulsation theory. There is no previous observational evidence or theoretical support for regular pulsations with periods as long as $11-13$ hours in any hot subdwarf. Hence, although pulsation remains the preferred explanation for light variability in UVO $0825+15$, it is likely to remain contentious without additional evidence.

\section{CONCLUSION}

We have obtained NOT/ALFOSC and Subaru/HDS spectroscopy, and K2 photometry of the hot subdwarf UVO $0825+15$, which we have augmented with archival $I U E$ spectrophotometry and broad-band photometry.

NOT/ALFOSC spectroscopy rules out any short-period $(<7 \mathrm{~d})$ binary companion, and provides fundamental atmospheric parameters of $T_{\text {eff }}=38900 \pm 270 \mathrm{~K}, \log g / \mathrm{cm} \mathrm{s}^{-2}=$ $5.97 \pm 0.11$ and $\log n_{\mathrm{He}} / n_{\mathrm{H}}=-0.57 \pm 0.01$ (formal errors). These results are supported by the IUE spectrophotometry which, in addition, give $E_{B-V} \approx 0.03$ and angular radius $\theta=1.062 \pm 0.006 \times 10^{-11}$ radians.

Subaru/HDS spectroscopy provides elemental abundances in the stellar atmosphere for 14 species including yttrium, germanium and lead, and upper limits for three, including carbon and iron. Carbon is at least 2 dex sub-solar and iron is approximately solar. Otherwise all measured elements heavier than argon are $\approx 2-4$ dex overabundant, whilst light elements from nitrogen to sulphur are solar or sub-solar. UVO $0825+15$ thus forms the fourth member of a group of "heavy metal" hot subdwarfs; these have intermediate helium-rich atmospheres and 3 to 4 dex overabundances of one or more of germanium, strontium, yttrium, zirconium, and lead. The peculiar chemistry probably arises as a result of radiative levitation producing a heavily stratified atmosphere with overabundant species having a high specific opacity at the local temperature of the line-forming region. Other interpretations are possible, but unlikely. Even when the chemistry is understood, assessing the evolutionary origin of UVO $0825+15$ will present a significant challenge.

Over 150 unidentified absorption lines in the $S u b$ aru/HDS spectrum present another challenge, possibly leading to the identification of additional species. Other heavymetal subdwarfs analyzed so far are essentially free of this problem. A high-resolution ultraviolet spectrum is well within reach of this UV-bright star using the Hubble Space Telescope and would provide crucial information about species not seen in the optical, about stratification in the photosphere, and about the opacity required to model the photosphere correctly.

K2 photometry reveals a unique light curve having a dominant period around $10.8 \mathrm{~h}$, but with a semi-amplitude which varies from $1-5 \mathrm{ppt}$ over the $75 \mathrm{~d}$ of observa- tion. The first harmonic of the $10.8 \mathrm{~h}$ period, and another period at $13.3 \mathrm{~h}$ are also present for most of the light curve. The periods are not constant throughout the observations, and may be affected by additional unresolved components. The favoured explanation for the light variability in UVO $0825+15$ is a multi-periodic non-radial oscillation due to g-modes with very high radial order. However, the periods are some 300 times longer than the fundamental radial mode for such stars, and no theory predicts such modes to exist. Alternative explanations involving a stellar mass (or other) companion fail for lack of radial-velocity evidence. High-precision $\left(\sim 1 \mathrm{~km} \mathrm{~s}^{-1}\right)$ radial-velocity studies over a 12 hour interval would be instructive.

Taking the extraordinary combination of observed properties and the associated difficulties in interpretation, UVO $0825+15$ presents one of the most challenging hot subdwarfs known to date and is a strong candidate to be the first "pulsating lead-rich hot subdwarf".

\section{ACKNOWLEDGMENTS}

Research at the Armagh Observatory and Planetarium is supported by a grant-in-aid from the Northern Ireland Department for Communities. CSJ acknowledges support from the UK Science and Technology Facilities Council (STFC) Grant No. ST/M000834/1. HPP acknowledges support from STFC Grant No. ST/M502268/1. ASB gratefully acknowledges financial support from the Polish National Science Centre under project No. UMO2011/03/D/ST9/01914. This paper is based upon work supported by the National Auronautics and Space Adminstration under Grant NNH14ZDA001n-K2GO1. Funding for this research was provided by the National Science Foudation (USA) grant \#1312869.

This paper includes data collected by the Kepler mission. Funding for the Kepler mission is provided by the NASA Science Mission directorate.

This paper is based in part on data collected at Subaru Telescope, which is operated by the National Astronomical Observatory of Japan.

Based on observations made with the Nordic Optical Telescope, operated by the Nordic Optical Telescope Scientific Association at the Observatorio del Roque de los Muchachos, La Palma, Spain, of the Instituto de Astrofisica de Canarias.

Some of the data presented in this paper were obtained from the Mikulski Archive for Space Telescopes (MAST). STScI is operated by the Association of Universities for Research in Astronomy, Inc., under NASA contract NAS526555. Support for MAST for non-HST data is provided by the NASA Office of Space Science via grant NNX09AF08G and by other grants and contracts.

This research has made use of the SIMBAD database, operated at CDS, Strasbourg, France.

This work has made use of the Vienna Atomic Line Database (VALD) database, operated at Uppsala University, the Institute of Astronomy of the Russian Academy of Sciences in Moscow, and the University of Vienna, the Atomic Line List, hosted by the Department of Physics and Astronomy, University of Kentucky, and the National Insti- 
tute of Standards and Technology (NIST) Atomic Spectra Database, which is hosted by the U.S. Dept of Commerce.

The TheoSSA TMAP service (http://dc.gvo.org/theossa) used to retrieve theoretical spectra for this paper was constructed as part of the activities of the German Astrophysical Virtual Observatory.

This research has made use of IRAF, the Image Reduction and Analysis Facility, written and supported by the National Optical Astronomy Observatories (NOAO) in Tucson, Arizona. NOAO is operated by the Association of Universities for Research in Astronomy (AURA), Inc. under cooperative agreement with the National Science Foundation.

This research has made use of the period-analysis software Ts (Templeton 2004) made available by the AAVSO, Cambridge, Massachusetts, USA.

The authors are grateful to Thomas Rauch for assistance with running TMAP, to Philip Hall for providing the zero-age helium main-sequence data for Fig. 7, and to the referee for drawing attention to numerous details which deserved correction or improvement.

\section{REFERENCES}

Ahmad A., Jeffery C. S., 2003, A\&A, 402, 335

Ahmad A., Jeffery C. S., 2005, A\&A, 437, L51

Ahmad A., Behara N. T., Jeffery C. S., Sahin T., Woolf V. M., 2007, A\&A, 465, 541

Aoiki W., 2008, "Data reduction of échelle spectra with IRAF": http://www.subarutelescope.org/Observing/Instruments/HDS/specana200810e.pdf

Asplund M., Grevesse N., Sauval A. J., Scott P., 2009, ARA\&A, 47,481

Bear E., Soker N., 2014, MNRAS, 437, 1400

Beauchamp A., Wesemael F., Bergeron P., 1997, ApJS, 108, 559

Becker S. R., Butler K., 1989, A\&A, 209, 244

Becker S. R., Butler K., 1990, A\&A, 235, 326

Behara N. T., Jeffery C. S., 2006, A\&A, 451, 643

Bell K. L., Hibbert A., Stafford R. P., McLaughlin B. M., 1994, Phys. Scr., 50, 343

Berger J., Fringant A.-M., 1980, A\&A, 85, 367

Brown T. M., Landsman W. B., Randall S. K., Sweigart A. V., Lanz T., 2013, ApJ, 777, L22

Butler K., 1984, PhD Thesis, University College London

Carnochan D. J., Wilson R., 1983, MNRAS, 202, 317

Cunto W., Mendoza C., Ochsenbein F., Zeippen C. J., 1993, A\&A, 275, L5

Cutri R. M., et al., 2003, VizieR Online Data Catalog, 2246

Dorman B., Rood R. T., O'Connell R. W., 1993, ApJ, 419, 596

Edelmann H., Heber U., Hagen H.-J., Lemke M., Dreizler S., Napiwotzki R., Engels D., 2003, A\&A, 400, 939

Fontaine G., Brassard P., Green E. M., Chayer P., Charpinet S., Andersen M., Portouw J., 2008, A\&A, 486, L39

Geier S., 2013, å, 549, A110

Green E. M., et al., 2011, ApJ, 734, 59

Hansen C. J., Winget D. E., Kawaler S. D., 1985, ApJ, 297, 544

Hardorp J., Scholz M., 1970, ApJS, 19, 193

Heber U., 2016, PASP

Høg E., et al., 2000, A\&A, 355, L27

Hummer D. G., Berrington K. A., Eissner W., Pradhan A. K., Saraph H. E., Tully J. A., 1993, A\&A, 279, 298

Jeffery C. S., 1991, Newsletter on 'Analysis of Astronomical Spectra', 16,17

Jeffery C. S., 2011, Information Bulletin on Variable Stars, 5964,

Jeffery C. S., Saio H., 2006, MNRAS, 372, L48
Jeffery C. S., Saio H., 2016, MNRAS, 458, 1352

Jeffery C. S., Hamill P. J., Harrison P. M., Jeffers S. V., 1998, A\&A, 340, 476

Jeffery C. S., Starling R. L. C., Hill P. W., Pollacco D., 2001, MNRAS, 321, 111

Jeffery C. S., et al., 2013, MNRAS, 429, 3207

Kilkenny D., 1988, MNRAS, 232, 377

Koen C., Kilkenny D., O'Donoghue D., van Wyk F., Stobie R. S., 1997, MNRAS, 285, 645

Kramida A., Ralchenko Y., Reader J., NIST ASD Team. 2015, Technical report, NIST Atomic Spectra Database. National Institute of Standards and Technology, Gaithersburg, MD.

Kurucz R. L., 1999, Robert L. Kurucz on-line database of observed and predicted atomic transitions

Kurucz R., Bell B., 1995, Atomic Line Data (R.L. Kurucz and B. Bell) Kurucz CD-ROM No. 23. Cambridge, Mass.: Smithsonian Astrophysical Observatory, 1995., 23

Latour M., Fontaine G., Brassard P., Green E. M., Chayer P., Randall S. K., 2011, ApJ, 733, 100

Martin G., Fuhr J., Wiese W., 1988, J. Phys. Chem. Ref. Data Suppl., 17

Martin P., Jeffery C. S., Naslim N., Woolf V. M., 2016, MNRAS, submitted

McNamara B. J., Jackiewicz J., McKeever J., 2012, AJ, 143, 101

Miller Bertolami M. M., Córsico A. H., Althaus L. G., 2011, ApJ, $741, \mathrm{~L} 3+$

Naslim N., Jeffery C. S., Ahmad A., Behara N. T., Şahìn T., 2010, MNRAS, 409, 582

Naslim N., Jeffery C. S., Behara N. T., Hibbert A., 2011, MNRAS, 412,363

Naslim N., Geier S., Jeffery C. S., Behara N. T., Woolf V. M., Classen L., 2012, MNRAS, 423, 3031

Naslim N., Jeffery C. S., Hibbert A., Behara N. T., 2013, MNRAS, 434, 1920

Németh P., Kawka A., Vennes S., 2012, MNRAS, 427, 2180

Noguchi K., et al., 2002, PASJ, 54, 855

O'Toole S. J., Heber U., 2006, A\&A, 452, 579

Østensen R. H., 2012, in Kilkenny D., Jeffery C. S., Koen C., eds, ASPCS Vol. 452, The Fifth Meeting on Hot Subdwarf Stars and Related Objects. pp 233-240

Østensen R. H., et al., 2010, MNRAS, 409, 1470

Østensen R. H., et al., 2012, ApJ, 753, L17

Piskunov N. E., Kupka F., Ryabchikova T. A., Weiss W. W., Jeffery C. S., 1995, A\&AS, 112, 525

Press W. H., Teukolsky S. A., Vetterling W. T., Flannery B. P., 1989, Numerical Recipes: The Art of Scientific Computing (FORTRAN version). Cambridge University Press, New York, NY, USA

Randall S. K., Bagnulo S., Ziegerer E., Geier S., Fontaine G., 2015, A\&A, 576, A65

Randall S. K., et al., 2016, A\&A, 589, A1

Ringat E., Rauch T., Werner K., 2012, Baltic Astronomy, 21, 341

Ryabchikova T., Piskunov N., Kurucz R. L., Stempels H. C., Heiter U., Pakhomov Y., Barklem P. S., 2015, Phys. Scr., 90, 054005

Schöning T., Butler K., 1989, A\&AS, 78, 51

Silvotti R., et al., 2014, A\&A, 570, A130

Templeton M., 2004, Journal of the American Association of Variable Star Observers (JAAVSO), 32, 41

Thompson G. I., Nandy K., Jamar C., Monfils A., Houziaux L., Carnochan D. J., Wilson R., 1978, Catalogue of stellar ultraviolet fluxes. A compilation of absolute stellar fluxes measured by the Sky Survey Telescope (S2/68) aboard the ESRO satellite TD-1. The Science Research Council

Townsend R. H. D., MacDonald J., 2006, MNRAS, 368, L57

Tujitsu A., 2013, "Quick Reduction of HDS data on IRAF": http://www.subarutelescope.org/Observing/Instruments/HDS/hdsql-e.html 
Vennes S., Kawka A., Németh P., 2011, MNRAS, 410, 2095

Vidal C. R., Cooper J., Smith E. W., 1973, ApJS, 25, 37

Warner B., Kirkpatrick R., 1969, MNRAS, 144, 397

Wenger M., et al., 2000, A\&AS, 143, 9

Werner K., Dreizler S., Rauch T., 2012, TMAP: Tübingen NLTE Model-Atmosphere Package, Astrophysics Source Code Library (ascl:1212.015)

Wiese W. L., Smith M. W., Glennon B. M., 1966, Atomic transition probabilities. Vol.: Hydrogen through Neon. A critical data compilation

Wiese W. L., Smith M. W., Miles B. M., 1969, Atomic transition probabilities. Vol. 2: Sodium through Calcium. A critical data compilation

Woolf V. M., Jeffery C. S., 2002, A\&A, 395, 535

Woudt P. A., et al., 2006, MNRAS, 371, 1497

Zacharias N., et al., 2009, VizieR Online Data Catalog, 1315

The Opacity Project Team 1995, The Opacity Project Vol. 1. Institute of Physics Publications, Bristol, UK

\section{APPENDIX A: SPECTRAL ATLAS AND LINE ABUNDANCES FOR UVO 0825+15}

Figures A.1 to A.8 contain an atlas of the Subaru/HDS spectrum of UVO $0825+15$, with the best model fit and identifications of absorption lines. Table A.1 shows equivalent widths, adopted transition probabilities, and derived abundances for individual lines in the above spectrum.
Table A.1. Equivalent widths and scaled abundances $\log \epsilon$ for identified unblended absorption lines. The sources of oscillator strengths $(g f)$ are given with the ion designation. Abundances were computed assuming a model atmosphere with $T_{\text {eff }}=$ $39000 \mathrm{~K}, \log g / \mathrm{cm} \mathrm{s}^{-2}=6.0, v_{\mathrm{t}}=2 \mathrm{~km} \mathrm{~s}^{-1}$, and composition as in Table 2. Errors on the mean abundance per ion are given as the standard deviation where the number of lines $n>2$, the semirange where $n=2$ and adopting the mean measurement error of $\pm 2 \mathrm{~m} \AA$ where $n=1$.

\begin{tabular}{|c|c|c|c|}
\hline $\begin{array}{l}\text { Ion } \\
\lambda / \AA\end{array}$ & $\begin{array}{r}\text { Reference } \\
\log g f\end{array}$ & $W_{\lambda} / \mathrm{m} \AA$ & $\log \epsilon$ \\
\hline $\mathrm{N}_{\text {II }}$ & \multicolumn{3}{|c|}{ Becker \& Butler (1989) } \\
\hline 3919.01 & -0.335 & 8 & 8.13 \\
\hline 3995.00 & 0.225 & 25 & 7.88 \\
\hline 4171.60 & 0.280 & 7 & 7.87 \\
\hline 4175.66 & -1.180 & 11 & 7.79 \\
\hline 4241.18 & -0.336 & 29 & 8.01 \\
\hline 4431.82 & -0.152 & 12 & 7.80 \\
\hline 4432.74 & 0.595 & 7 & 7.54 \\
\hline 4447.03 & 0.238 & 23 & 8.14 \\
\hline 4530.40 & 0.671 & 19 & 8.07 \\
\hline 4552.53 & 0.207 & 19 & 8.52 \\
\hline 4601.48 & -0.385 & 13 & 8.18 \\
\hline 4607.16 & -0.483 & 7 & 8.00 \\
\hline 4613.87 & -0.607 & 14 & 8.45 \\
\hline 4630.54 & 0.093 & 23 & 8.00 \\
\hline 4643.09 & -0.385 & 13 & 8.18 \\
\hline 5001.13 & 0.282 & 17 & 7.60 \\
\hline 5005.15 & 0.612 & 24 & 7.89 \\
\hline 5007.33 & 0.161 & 18 & 8.21 \\
\hline \multirow[t]{2}{*}{5045.09} & -0.389 & 7 & 7.89 \\
\hline & & & $8.01 \pm 0.25$ \\
\hline N III & \multicolumn{3}{|c|}{ Butler (1984) } \\
\hline 3745.95 & -0.780 & 8 & 8.03 \\
\hline 3754.69 & -0.480 & 17 & 8.14 \\
\hline 3771.03 & -0.300 & 16 & 7.93 \\
\hline 4544.80 & -0.143 & 17 & 8.33 \\
\hline 4546.32 & 0.017 & 18 & 8.32 \\
\hline \multirow[t]{2}{*}{4641.85} & \multirow[t]{2}{*}{-0.815} & \multirow[t]{2}{*}{29} & 8.20 \\
\hline & & & $8.16 \pm 0.16$ \\
\hline O II & \multirow{3}{*}{\multicolumn{2}{|c|}{$\begin{array}{l}\text { Bell et al. (1994) } \\
0.308\end{array}$}} & \\
\hline \multirow[t]{2}{*}{4649.14} & & & 7.47 \\
\hline & & & $7.47 \pm 0.09$ \\
\hline \multicolumn{4}{|l|}{ O III } \\
\hline \multirow[t]{2}{*}{3754.70} & \multirow[t]{2}{*}{-0.099} & \multirow[t]{2}{*}{12} & 7.38 \\
\hline & & & $7.38 \pm 0.07$ \\
\hline NeII & \multicolumn{2}{|c|}{ Wiese et al. (1966) } & \\
\hline 3664.07 & -0.260 & 16 & 7.75 \\
\hline 3709.62 & -0.330 & 7 & 7.44 \\
\hline \multirow[t]{2}{*}{3713.08} & \multirow[t]{2}{*}{0.260} & \multirow[t]{2}{*}{14} & 7.26 \\
\hline & & & $7.48 \pm 0.25$ \\
\hline \multirow{3}{*}{$\begin{array}{l}\mathrm{Mg} \text { II } \\
4481.13\end{array}$} & \multicolumn{2}{|c|}{ Wiese et al. (1969) } & \\
\hline & 0.568 & 7 & 6.25 \\
\hline & & & $6.25 \pm 0.11$ \\
\hline \multirow{3}{*}{$\begin{array}{l}\text { Al III } \\
3601.63\end{array}$} & \multicolumn{2}{|c|}{ Cunto et al. (1993) } & \\
\hline & 0.000 & 12 & 6.47 \\
\hline & & & $6.47 \pm 0.07$ \\
\hline
\end{tabular}


Table A.1. (cont.)

\begin{tabular}{|c|c|c|c|}
\hline $\begin{array}{l}\text { Ion } \\
\lambda / \AA\end{array}$ & $\begin{array}{r}\text { Reference } \\
\log g f\end{array}$ & $W_{\lambda} / \mathrm{m} \AA$ & $\log \epsilon$ \\
\hline Si IV & \multicolumn{3}{|c|}{ Becker \& Butler (1990) } \\
\hline 3762.43 & 0.250 & 22 & 6.57 \\
\hline 3773.15 & -0.010 & 6 & 6.28 \\
\hline 4088.85 & 0.199 & 37 & 6.14 \\
\hline 4116.10 & -0.103 & 32 & 6.33 \\
\hline 4631.38 & 1.217 & 6 & 5.94 \\
\hline \multirow[t]{2}{*}{4654.14} & 1.486 & 24 & 6.31 \\
\hline & & & $6.26 \pm 0.21$ \\
\hline S III & \multicolumn{3}{|c|}{ Hardorp \& Scholz (1970) } \\
\hline 3656.60 & -0.830 & 13 & 7.66 \\
\hline 3662.01 & -0.380 & 25 & 7.56 \\
\hline 3717.77 & -0.190 & 42 & 7.65 \\
\hline 3778.90 & -0.290 & 18 & 7.28 \\
\hline 3837.80 & -0.570 & 21 & 7.65 \\
\hline 3928.59 & -0.190 & 43 & 7.70 \\
\hline 3961.56 & -0.810 & 11 & 7.58 \\
\hline 3983.77 & -0.720 & 26 & 7.94 \\
\hline 4253.59 & 0.400 & 70 & 7.42 \\
\hline 4284.98 & 0.110 & 50 & 7.48 \\
\hline \multirow[t]{2}{*}{4332.69} & -0.240 & 50 & 7.83 \\
\hline & & & $7.61 \pm 0.18$ \\
\hline Cl III & \multicolumn{3}{|c|}{ Wiese et al. (1969) } \\
\hline \multirow[t]{2}{*}{3720.45} & 0.350 & 7 & 6.34 \\
\hline & & & $6.34 \pm 0.11$ \\
\hline Ti III & Warner \& & Kirkpatrick & (1969) \\
\hline 3915.26 & 0.066 & 14 & 8.01 \\
\hline \multirow[t]{2}{*}{4214.93} & -0.189 & 9 & 8.06 \\
\hline & & & $8.03 \pm 0.03$ \\
\hline Ti IV & Kurucz (19 & 99) & \\
\hline 4131.26 & 0.918 & 14 & 6.68 \\
\hline 4618.04 & 0.277 & 23 & 7.34 \\
\hline \multirow[t]{2}{*}{4677.59} & 0.339 & 12 & 7.38 \\
\hline & & & $7.13 \pm 0.39$ \\
\hline V IV & Martin et & 1. $(1988)$ & \\
\hline 4841.26 & 0.150 & 7 & 7.16 \\
\hline 4906.29 & 0.300 & 19 & 7.57 \\
\hline 4985.64 & 0.520 & 21 & 7.45 \\
\hline 5130.78 & 0.620 & 38 & 7.85 \\
\hline \multirow[t]{2}{*}{5146.52} & 0.410 & 20 & 7.50 \\
\hline & & & $7.51 \pm 0.25$ \\
\hline & Naslim et & 1. $(2011)$ & \\
\hline \multirow[t]{2}{*}{4178.96} & 0.341 & 19 & 6.24 \\
\hline & & & $6.24 \pm 0.04$ \\
\hline Y III & Naslim et & 1. $(2011)$ & \\
\hline 4039.600 & 1.005 & 8 & 5.47 \\
\hline \multirow[t]{2}{*}{4040.110} & 1.005 & 6 & 5.28 \\
\hline & & & $5.37 \pm 0.09$ \\
\hline $\mathrm{Pb}$ IV & Naslim et & 1. $(2013)$ & \\
\hline 3962.48 & -0.025 & 12 & 5.30 \\
\hline 4049.80 & -0.010 & 24 & 5.66 \\
\hline \multirow[t]{2}{*}{4496.15} & -0.237 & 10 & 5.51 \\
\hline & & & $5.49 \pm 0.18$ \\
\hline
\end{tabular}

Table A.1. (cont.)

\begin{tabular}{lcrl}
\hline Ion & Reference & & \\
$\lambda / \AA$ & $\log g f$ & $W_{\lambda} / \mathrm{m} \AA$ & $\log \epsilon$ \\
\hline Ca II & Wiese et al. $(1969)$ & \\
3706.02 & -0.441 & 6 & 8.15 \\
3933.66 & 0.134 & 51 & 7.95 \\
\cline { 3 - 4 } & & & $8.05 \pm 0.10$ \\
\cline { 3 - 4 } Ca III & Kurucz $(1999)$ & & \\
3706.02 & -0.441 & 6 & 8.15 \\
3761.61 & -1.364 & 18 & 7.95 \\
3949.61 & -0.748 & 8 & 8.40 \\
4136.25 & 0.166 & 22 & 8.47 \\
4136.25 & 0.166 & 9 & 7.94 \\
4153.57 & -0.334 & 22 & 8.54 \\
4164.30 & 0.258 & 26 & 8.36 \\
4175.65 & -0.359 & 17 & 8.44 \\
4184.20 & -0.045 & 21 & 8.23 \\
4211.61 & -0.506 & 6 & 8.41 \\
4213.13 & -0.394 & 13 & 8.27 \\
4233.71 & -1.092 & 45 & 8.50 \\
4256.65 & -0.473 & 7 & 8.43 \\
4271.82 & -0.919 & 25 & 8.25 \\
4273.88 & -0.654 & 14 & 8.63 \\
4278.22 & -0.369 & 12 & 8.26 \\
4278.82 & -1.028 & 29 & 8.32 \\
4283.56 & -2.455 & 33 & 8.31 \\
4301.01 & 0.401 & 33 & 8.62 \\
4431.29 & 0.273 & 19 & 7.92 \\
4462.47 & 0.120 & 7 & 8.11 \\
4484.40 & -0.828 & 7 & 8.54 \\
4499.88 & 0.472 & 31 & 8.18 \\
4516.59 & 0.287 & 27 & 8.24 \\
4553.29 & 0.049 & 16 & 8.30 \\
4716.29 & -0.439 & 9 & 8.26 \\
4736.67 & -0.116 & 9 & 8.26 \\
4889.82 & -0.232 & 17 & 8.75 \\
4899.31 & -0.322 & 5 & 8.17 \\
4919.28 & 0.057 & 30 & 8.79 \\
5046.92 & -0.002 & 15 & 8.44 \\
5050.09 & -0.290 & 10 & 8.26 \\
5112.98 & -0.123 & 8 & 8.20 \\
5137.73 & 0.086 & 11 & 8.20 \\
\cline { 3 - 4 } & & & $8.32 \pm 0.21$ \\
\hline & & &
\end{tabular}



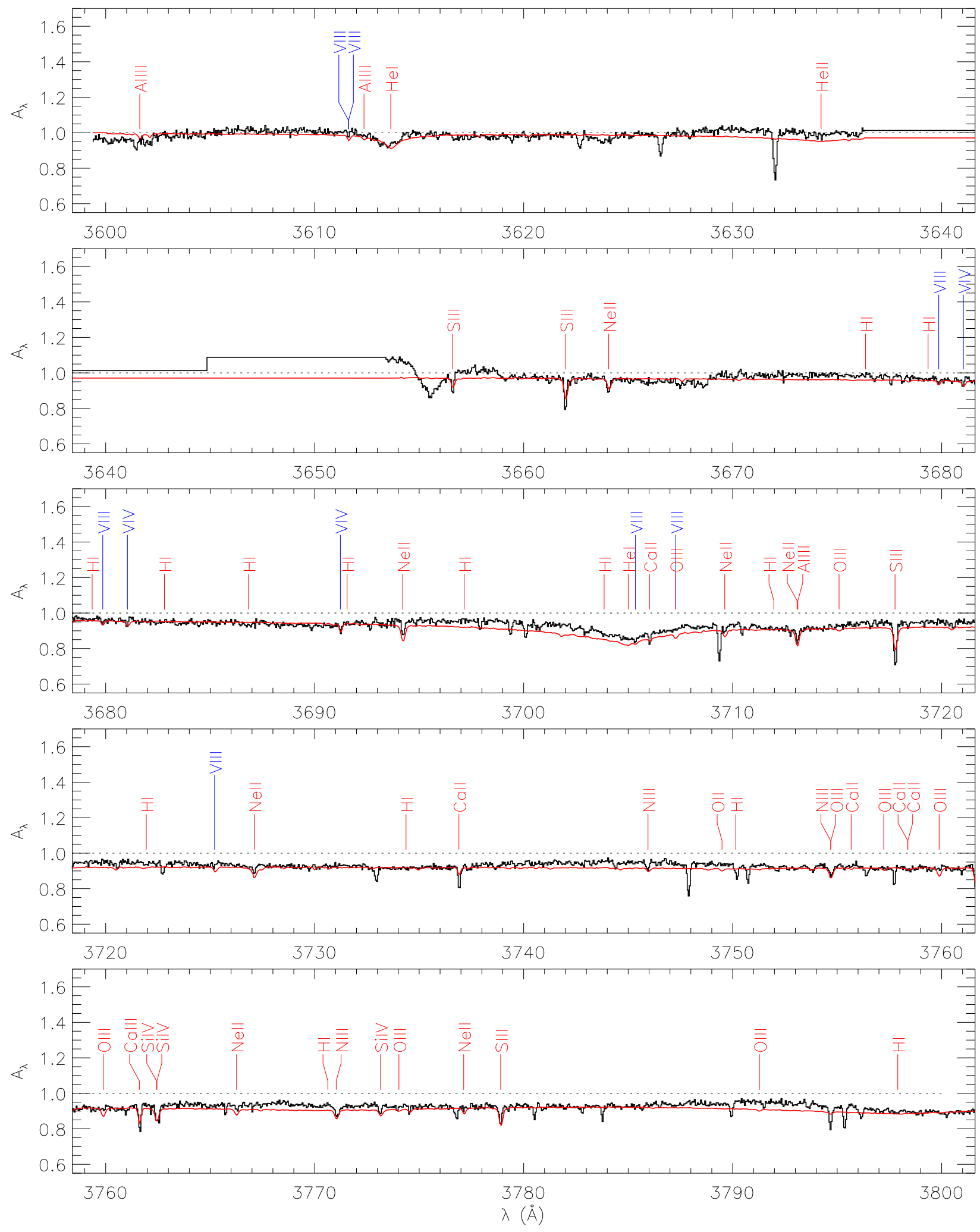

Figure A.1. Subaru/HDS spectrum of UVO $0825+15$ (black histogram), and best-fit model having $T_{\text {eff }}=39000 \mathrm{~K}, \log g / \mathrm{cm} \mathrm{s}^{-2}=6.0$ ), $n_{\mathrm{He}} / n_{\mathrm{H}}=0.25$ and abundances shown in Table 2. Lines with theoretical equivalent widths greater than $5 \mathrm{~m} \AA$ are identified wherever possible, Gaps in the observed spectrum correspond to major instrumental artefacts. 

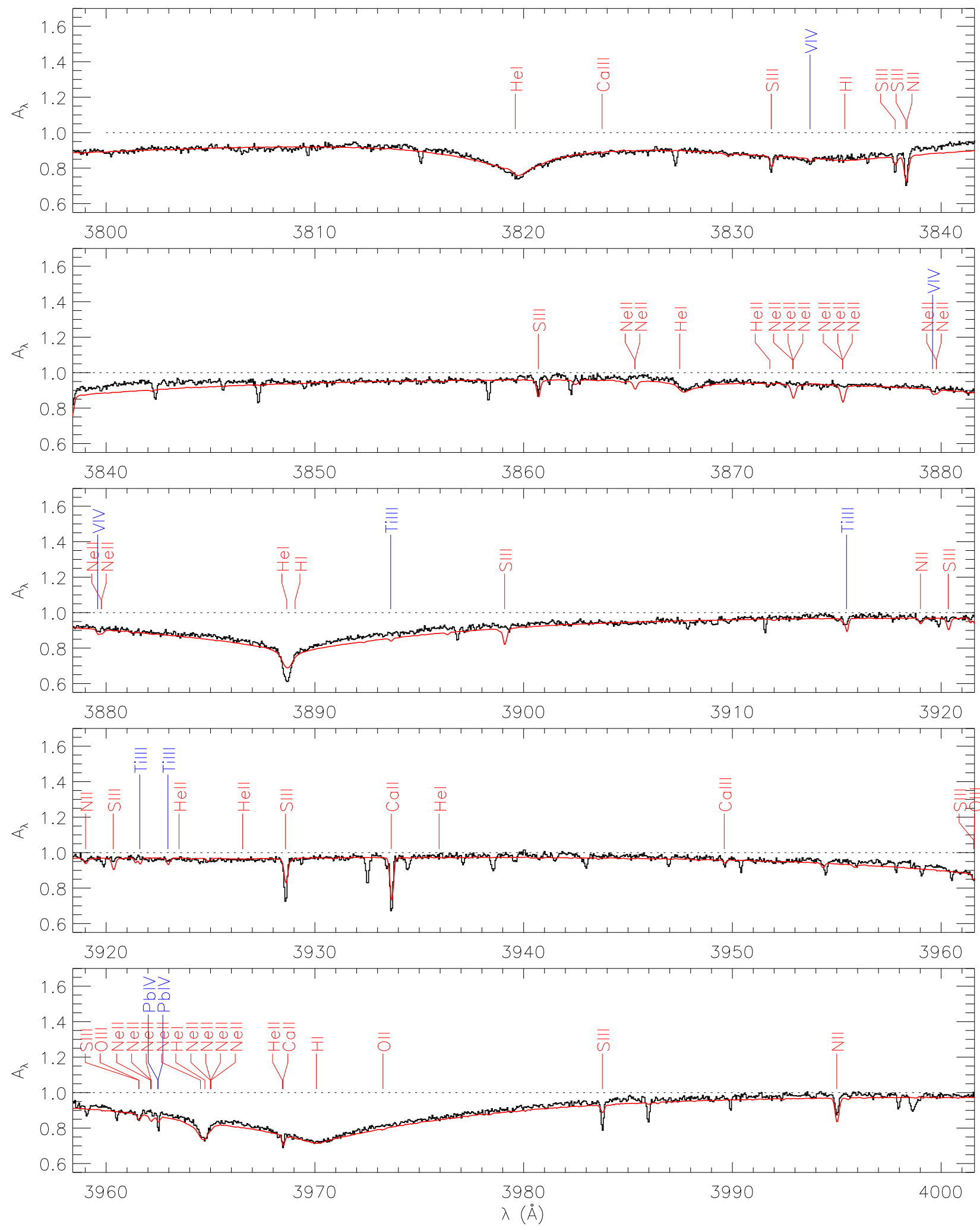

Figure A.2. As Fig. A.1 (contd.) 

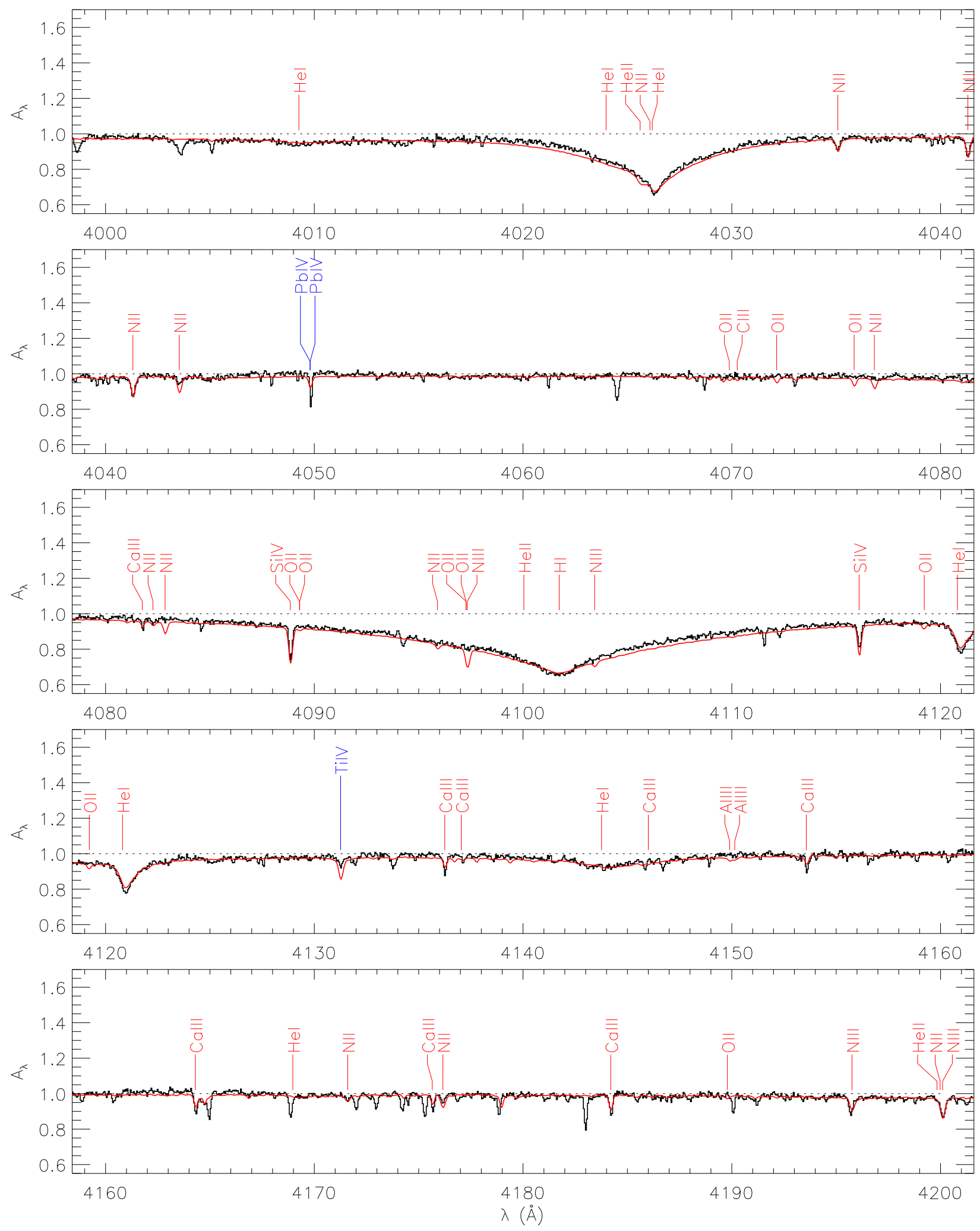

Figure A.3. As Fig. A.1 (contd.) 

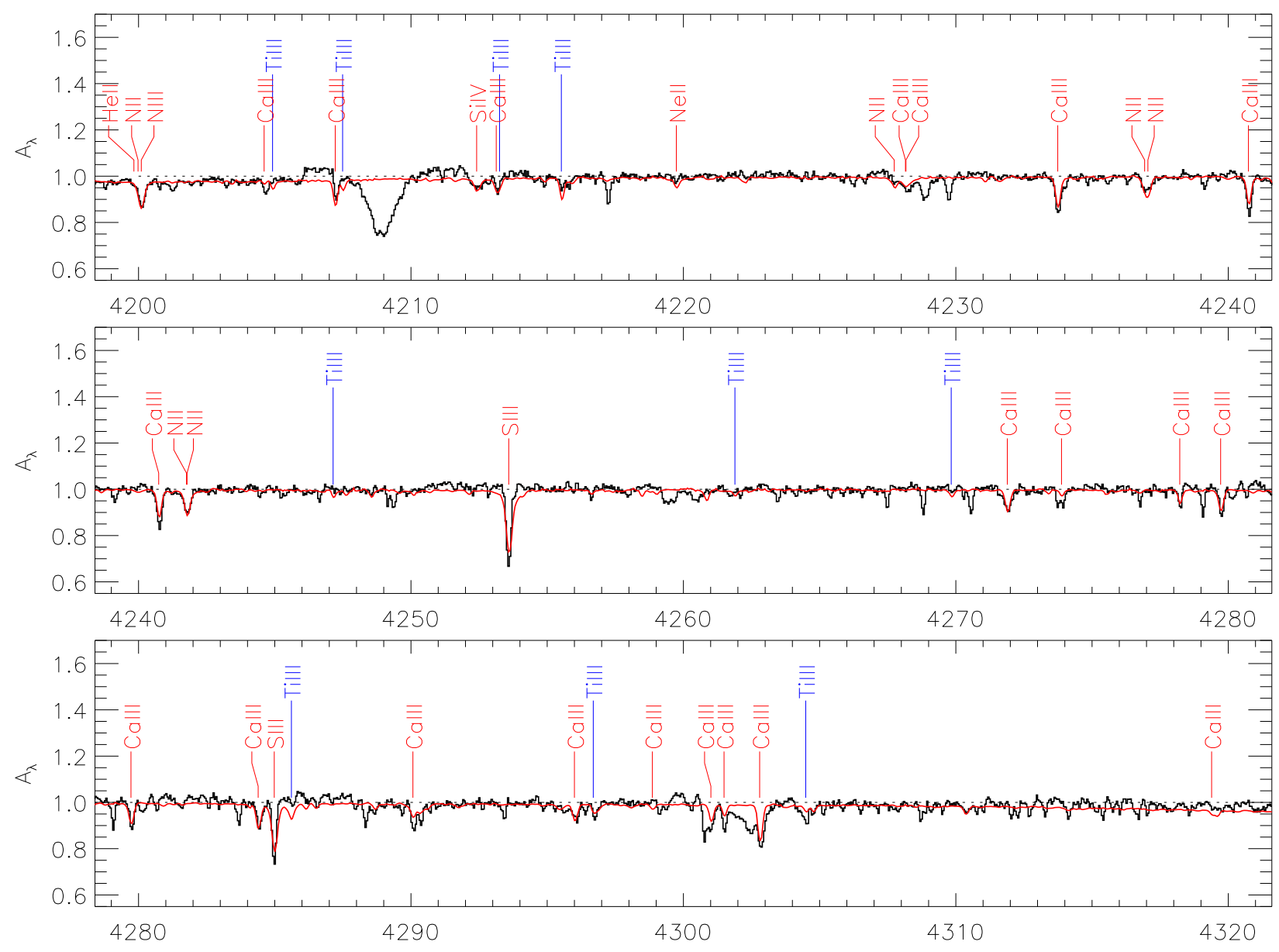

Figure A.4. As Fig. A.1 (contd.) 

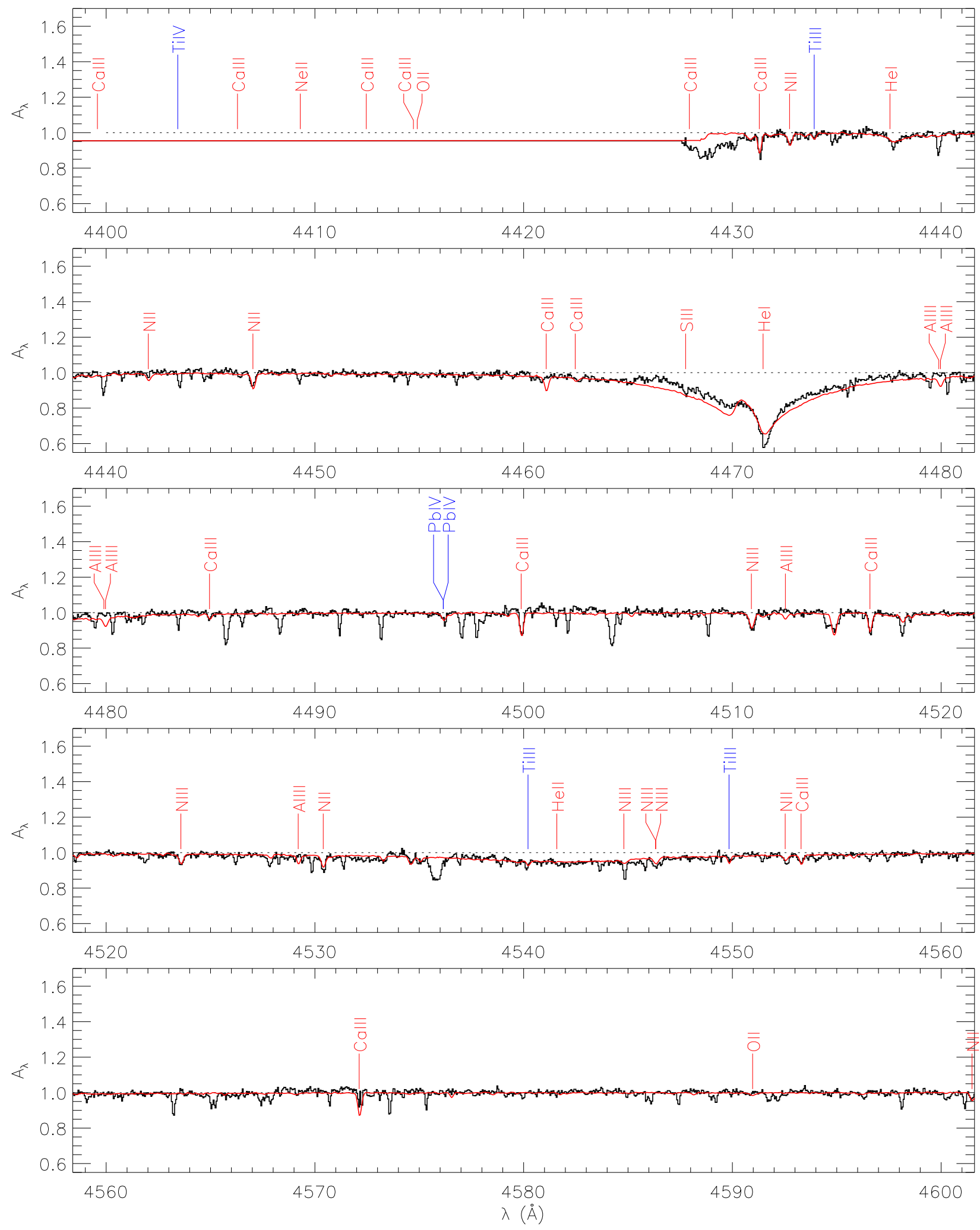

Figure A.5. As Fig. A.1 (contd.) 

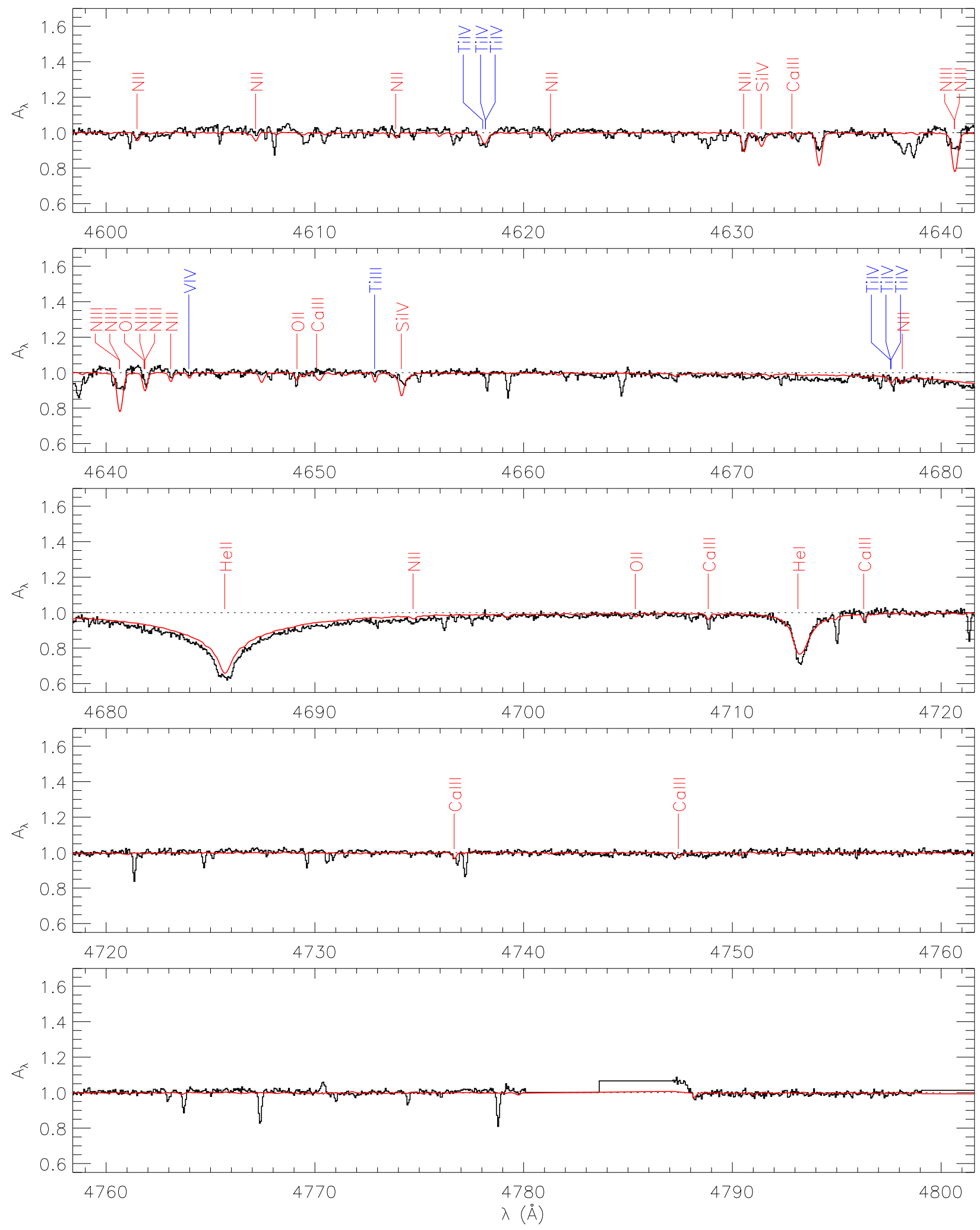

Figure A.6. As Fig. A.1 (contd.) 

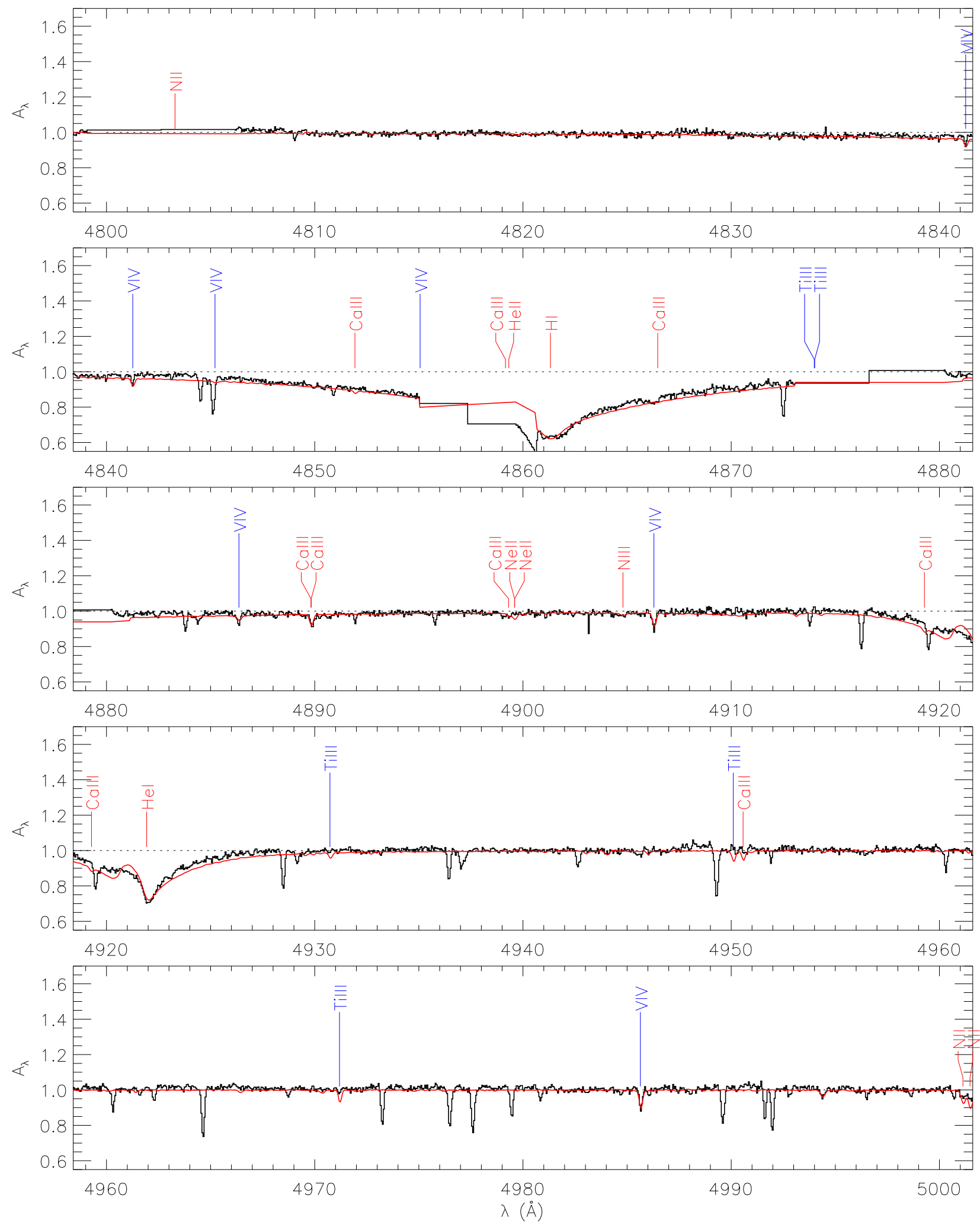

Figure A.7. As Fig. A.1 (contd.) 

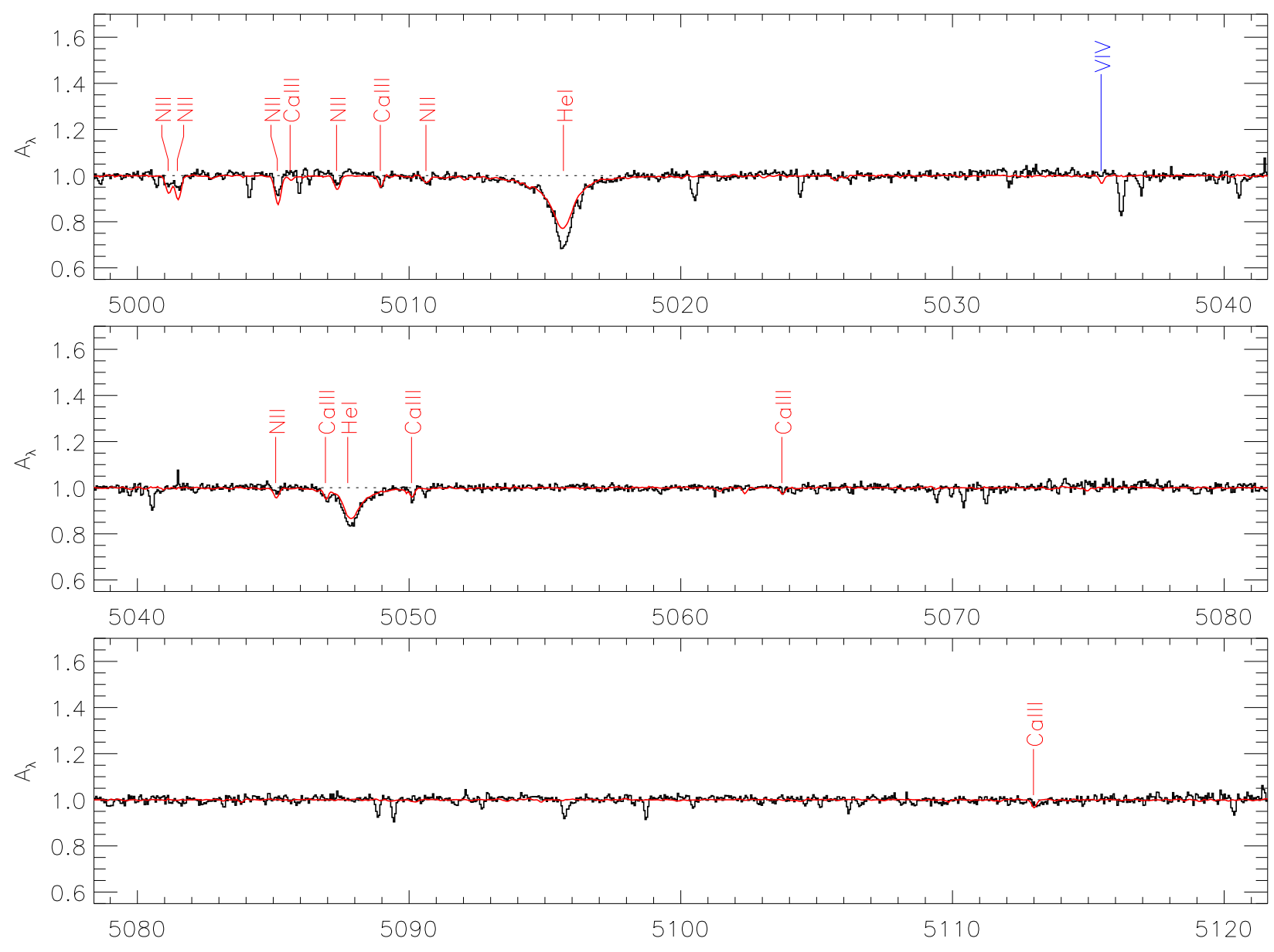

Figure A.8. As Fig. A.1 (contd.) 